\title{
Some Pairwise Balanced Designs
}

\author{
Malcolm Greig \\ Greig Consulting \\ 207-170 East Fifth St. \\ North Vancouver \\ BC, Canada, V7L 4L4 \\ greig@sfu.ca
}

Submitted: Oct. 6, 1998;

Accepted: Oct. 23, 1999.

\begin{abstract}
A pairwise balanced design, $B(K ; v)$, is a block design on $v$ points, with block sizes taken from $K$, and with every pair of points occurring in a unique block; for a fixed $K, B(K)$ is the set of all $v$ for which a $B(K ; v)$ exists.

A set, $S$, is a PBD-basis for the set, $T$, if $T=B(S)$. Let $N_{a(m)}=\{n: n \equiv$ $a \bmod m\}$, and $N_{\geq m}=\{n: n \geq m\}$; with $Q$ the corresponding restriction of $N$ to prime powers.

This paper addresses the existence of three PBD-basis sets.

1. It is shown that $Q_{1(8)}$ is a basis for $N_{1(8)} \backslash E$, where $E$ is a set of 5 definite and 117 possible exceptions.

2. We construct a 78 element basis for $N_{1(8)}$ with, at most, 64 inessential elements.

3. Bennett and Zhu have shown that $Q_{\geq 8}$ is a basis for $N_{\geq 8} \backslash E^{\prime}$, where $E^{\prime}$ is a set of 43 definite and 606 possible exceptions. Their result is improved to 48 definite and 470 possible exceptions. (Constructions for 35 of these possible exceptions are known.)

Finally, we provide brief details of some improvements and corrections to the generating/exception sets published in The CRC Handbook of Combinatorial Designs.
\end{abstract}

Key words and phrases: BIBD, Pairwise Balanced Design AMS subject classifications: Primary 05B05. 


\section{Introduction}

A pairwise balanced design, $B(K ; v)$, is a block design on $v$ points, with block sizes taken from $K$, and with every pair of points occurring in a unique block; for a fixed $K$, $B(K)$ is the set of all $v$ for which a $B(K ; v)$ exists.

A set, $S$, is a PBD-basis for the set, $T$, if $T=B(S)$. Let $N_{a(m)}=\{n: n \equiv a \bmod m\}$, and $N_{\geq m}=\{n: n \geq m\}$; with $Q$ the corresponding restriction of $N$ to prime powers.

This paper addresses the existence of three PBD-basis sets. The opening sections deal with useful known results and more general constructions. In Section 5, we give constructions that are particularly useful for the first of our two problems, then in Section 6 , we show that $Q_{1(8)}$ is a basis for $N_{1(8)} \backslash E$, where $E$ is a set of 5 definite and 117 possible exceptions; an application of this result is given in [5]. In Section 7, we construct a 78 element basis for $N_{1(8)}$ with, at least, 14 essential elements. In Section 8, we look at what $Q_{\geq 8}$ is a basis for. Bennett and Zhu have already tackled the last problem, and give some applications $[9,13]$; they have shown that $Q_{\geq 8}$ is a basis for $N_{\geq 8} \backslash E^{\prime}$, where $E^{\prime}$ is a set of 43 definite and 606 possible exceptions. Their result is improved to 48 definite and 470 possible exceptions. Results (without proofs) from this paper were incorporated into [12, Tables III.3.18-19].

Finally, in Section 9, we provide brief details of some improvements and corrections to the generating/exception sets published in The CRC Handbook of Combinatorial Designs, specifically [12, Tables III.3.17-19].

Although we sometimes give some non-existence results (phrased as definite exceptions and essential elements above) we will not establish these here; they require techniques quite different from the ones we are using here. We will merely note that most of them can be established by some results of Wilson's [41]: the integrality conditions, and the bound on the size of a flat; these conditions are also given in [12, Lemma 3.2, Theorem 3.1.2].

Remark 1.1 Wilson's integrality conditions imply that if $S \subset N_{1(m)}$, and if a $B(S ; v)$ exists, then it follows that $v \in N_{1(m)}$.

\section{Notation}

The notation we use is fairly straightforward. A $K$-GDD is a group divisible design with block sizes taken from $K$, and with a group size vector of $\left\{G_{1}, G_{2}, \ldots, G_{n}\right\}$; we will usually write the group size vector in exponential notation. We will only be concerned with the $\lambda=1$ case; i.e., a pair of elements from different groups occurs in one block of the design, whilst a pair of elements from the same group appears in no block.

A pairwise balanced block design is a GDD with a group size vector of $1^{v}$; it is denoted by $B(K ; v)$. If the block size is uniform, with $K=\{k\}$ then this block design is referred to as a balanced incomplete block design (a BIBD), and denoted by $B(k ; v)$.

If a $B(k ; v)$ exists, then the quantity $r=(v-1) /(k-1)$ is called the replication number of the BIBD, and we say that $r \in R N(k)$. 
A transversal design, $T D(k ; t)$, is a $\{k\}$-GDD of group type $t^{k}$.

The set of all $v$ for which a $B(K ; v)$ exists is denoted by $B(K)$, and a similar definition is used for $T D(k)$.

The notation $B\left(K \cup\left\{k^{*}\right\} ; v\right)$ indicates there is exactly one block of size $k$ in the design in addition to those of sizes from $K$ (further blocks of size $k$ can occur only if $k \in K)$.

Resolvable designs are designs that admit a partition of the block set into subsets of blocks that contain every point exactly once. These designs are denoted by the prefix $R$.

If a $R B(k ; v)$ exists, then we say that $r \in R R N(k)$, where $R R N(k) \subset R N(k)$.

Some notation that is specific to a section will be introduced in that section.

One final notational convention we have adopted should be mentioned. We have labelled several lemmas by a one or two letter code. This is summarized in the key in the appendix, which gives the symbolic parameters used in the lemma where the code is introduced, but numeric values are substituted in the table of constructions. This device allows considerable compression of the table, yet retains all the information needed to verify the constructions.

\section{Direct Constructions}

We start this section by summarizing some well-known results in the first lemma.

Lemma 3.1 If $q$ is a prime or prime power, then

1. $q^{2} \in R B(q)$, and

2. $q^{2}+q+1 \in B(q+1)$, and

3. $q^{3}+1 \in R B(q+1)$, and

4. $q^{3}+q^{2}+q+1 \in R B(q+1)$.

Proof: All these are standard results. The first two amount to the existence of the affine and projective geometries, $A G(2, q)$ and $P G(2, q)$. The resolvability of the unital design was shown by Bose (see $[15,26]$ ), and the resolvability of the last design can be found in Lorimer [33].

Lemma 3.2 Embedded in $P G\left(2,2^{N}\right)$ is a set of $v(n)=2^{n}\left(2^{n}-1\right) 2^{N-n}+2^{n}$ points whose incidences with the lines of the plane are all either 0 or $2^{n}$. Furthermore, if $m<n$, the set of $v(n)$ points contains a subset of $v(m)$ points whose incidence with the lines of the plane is either 0 or $2^{m}$.

Proof: The first part of this result was shown by Denniston [19]. Denniston considers an irreducible second order curve, $Q(x, y)=a x^{2}+b x y+c y^{2}$ over $G F\left(2^{N}\right)$ in the nonhomogeneous coordinates $(x, y)$ of the plane. Let $G$ be any additive sub-group of order 
$2^{n}$ of the additive group $G F\left(2^{N}\right)$. Then the set of points satisfying $Q(x, y) \subset G$ forms a Denniston arc, $\left\{v(n) ;\left\{0,2^{n}\right\}\right\}$, in $P G\left(2,2^{N}\right)$ with $v(n)=2^{N+n}-2^{N}+2^{n}$; note if we consider a sub-group $H$ of order $2^{m}$ and take $H \subset G$, this yields a $\left\{v(m) ;\left\{0,2^{m}\right\}\right\}$-arc whose points are a subset of the $v(n)$ points, so that Denniston arcs can be taken to be nested.

Corollary 3.3 If $k$ is a power of 2 , and $n$ is a non-negative integer, then $k(k-1) 2^{n}+k \in$ $R B(k)$.

Proof: The resolvability was demonstrated by Seiden in the case $n=1$, (see [39]), and her proof carries over to Denniston's arcs. The device used is to consider a line that has zero incidence with the arc; the incidence of its points with the non-zero lines of the arc identifies the resolution classes.

Corollary 3.4 The following designs exist:

1. $B\left(\left\{9,17^{*}\right\} ; 120+17=137=8 * 17+1\right)$;

2. $B\left(\left\{9,33^{*}\right\} ; 232+33=265=8 * 33+1\right)$;

3. $B\left(\left\{9,65^{*}\right\} ; 456+65=521=8 * 65+1\right)$;

4. $B\left(\left\{17,33^{*}\right\} ; 496+33=529=8 * 66+1\right)$;

5. $B(\{9,17\} ; 273-120=153=8 * 19+1)$;

6. $B(\{17,33\} ; 1057-496=561=8 * 70+1)$;

7. $B\left(\left\{9,17,33^{*}\right\} ; 496-232+33=297=8 * 37+1\right)$.

Proof: The first four examples are formed by adding a complete external line to the appropriate Denniston arc in planes of order 16, 32, 64 and 32. The next two designs are formed by taking the complements of the Denniston arcs used in parts 1 . and 4 . above. The final result comes from taking the difference of the two arcs of parts 2. and 4., in the plane of order 32 , again adding a complete external line.

Corollary 3.5 The following designs exist:

1. $B\left(\left\{8,9,16,17,27^{*}\right\} ; 291\right)$;

2. $B\left(\left\{8,9,16,17,31^{*}\right\} ; 295\right)$; 
Proof: Again we use the 264 point difference of two Denniston arcs that we used in Corollary 3.4.7, and now we add an incomplete external line.

If we have a projective plane, $P G(2, q)$, then we may generate a $T D(q+1 ; q)$ from it by deleting a point, and using the lines through that point to generate groups. Since all the Desarguesian planes have ovals, we may consider how these oval points are distributed amongst the groups. Recall that if $q$ is odd, then there are $q+1$ points in the oval; (if $D$ is the Singer difference set, then $-D$ is such an oval). If $q$ is even, with $D=2 D$, then we may augment this set, $-D$, of $q+1$ points by the point $\{0\}$ to get a set of $q+2$ hyperoval points, which we will also term an oval.

Lemma 3.6 If $q=2 t+1$ is an odd prime power, then we may form a TD $(q+1 ; q)$ such that the distribution of the oval points amongst the groups is of type $0^{1} 1^{q}$ or $0^{t} 1^{2} 2^{t}$ or $0^{t+1} 2^{t+1}$. If $q=2 t$ is an even prime power, then we may form a $T D(q+1 ; q)$ such that the distribution of the oval points amongst the groups is of type $1^{q+1}$ or $0^{t} 2^{t+1}$.

Proof: To demonstrate this in the odd case, we either delete an oval point, or a non-oval tangent point, or a non-tangent point, and note the number of external lines, tangents and secants that the deleted point lay on. For the plane of even order, we delete an oval point, or a non-oval point; there are no tangents here.

Lemma $3.7\{81,585\} \subset R B(9)$, and $\{73,433,577\} \subset B(9)$.

Proof: The first three values follow from Lemma 3.1 using $q=8$ or $q=9$. The values 433 and 577 result from constructions by Abel [1] and Buratti [17].

Lemma 3.8 If $t \in\{1,6,7\}$ or $t$ is a power of 2 , then $56 t+8 \in R B(8)$.

Proof: This follows from Lemma 3.1 using $q=8$ or $q=7$, or from Corollary 3.3 using $k=8$.

Lemma 3.9 If $q$ is a prime or prime power, and $q \equiv 1 \bmod 8$, and $q \notin\{25,89\}$ and $q<4096$, then $7 q+1 \in R B(8)$.

Proof: The results for $q \in\{9,17\}$ are given above. The remaining constructions were given by Greig [20]. It can be shown that the restriction $q<4096$ is unneccessary, although we do not need that improvement here.

Lemma 3.10 The $\{9\}-R G D D$ of type $3^{33}$ exists.

Proof: This design was constructed by Mathon; see [28].

The remaining basic designs we need are transversals. We will briefly summarize some well known results. 
Lemma 3.11 If $q$ is a prime or prime power, then $q \in T D(q+1)$.

Lemma 3.12 If $m \in T D(k+1)$, then $m \in T D(k)$.

Lemma 3.13 If $\{m, n\} \subset T D(k)$, then $m n \in T D(k)$.

These last three lemmas can be combined to give a weaker version of MacNeish's result [34].

Theorem 3.14 If $m$ has no prime divisors less than $k$, then $m \in T D(k+1)$.

Lemma 3.15 $T D(k+1)=R T D(k)$.

Lemma 3.16 If $t \neq 3$, then $8 t \in T D(9)$, and if $t \neq 4$, then $8 t+1 \in T D(9)$.

Proof: See [3]; for Wojtas' TD $(9 ; 48)$ see [18].

Unless otherwise noted, all the TDs we need are from [3]. The remaining designs we need are incomplete transversal designs (ITDs). Loosely speaking, an ITD, written as $T D(k ; m)-T D(k ; a)$, is a design that could be completed to a $T D(k ; m)$ by adding the blocks of a $T D(k ; a)$ to the ITD; we do not actually need to have a $T D(k ; a)$ to have an ITD. We have chosen to provide constructions for the ITDs we need, even though better values can sometimes be obtained from [4].

Lemma 3.17 If $m \in T D(k)$, then the incomplete transversal $T D(k ; m)-T D(k ; 1)$ exists.

Proof: Delete one block of the TD to get the ITD.

The other two constructions of ITDs that we use are corollaries of Wilson's basic construction [40].

Lemma 3.18 If $m \in T D(10)$ and $0 \leq n \leq m$, and $\{k, k+1\} \subset T D(9)$, then the incomplete transversal T $D(9 ; k m+n)-T D(9 ; n)$ exists.

Lemma 3.19 If $m \in T D(n)$ and $8 \leq n$, then the incomplete transversal $T D(9 ; 8 m+$ $n-8)-T D(9 ; n)$ exists.

\section{General Constructions}

Lemma 4.1 (Direct Product) If $m \in T D(n)$, then $m n \in B(\{m, n\})$.

Proof: We just fill in the groups of the TD. 
Theorem 4.2 Suppose we have a $K-G D D$ with $v$ points and a group size vector of $\left\{\left|G_{j}\right|: j=1, \ldots, g\right\}$, and, for the first $g-1$ groups, we have a $B\left(K \cup\left\{w^{*}\right\} ;\left|G_{j}\right|+w\right)$ with $0 \leq w$, and, for the last group we have a $B\left(K ;\left|G_{g}\right|+w\right)$; then there exists a $B(K ; v+w)$.

Proof: This is a standard result; we add $w$ infinite points, and when we fill the first $g-1$ groups, we align the $w$ block with the infinite points, and refrain from using it for these groups. Note that the resulting design does not contain a $w$ block, unless, possibly, when $w \in K$.

Corollary 4.3 (Indirect Product) If $(m-w) \in T D(n)$, and a $B(K ; m)$ containing a $B(K ; w)$ sub-design exists, (or a $B(K ; m)$ exists with $w=1)$, then $n(m-w)+w \in$ $B(K \cup\{n\})$.

Theorem 4.4 (Wilson's Fundamental Construction) Suppose there is a "master" $K-G D D$ with $g$ groups and a group size vector of $\left\{\left|G_{j}\right|: j=1, \ldots, g\right\}$, and a weighting that assigns a positive weight of $w(x)$ to each point $x$. Let $W\left(B_{i}\right)$ be the weight vector of the $i$-th block. If, for every block $B_{i}$, we have a $K^{\prime}-G D D$ with a group size vector of $W\left(B_{i}\right)$, then there exists a $K^{\prime}-G D D$ with a group size vector of $\left\{\sum_{x \in G_{j}} w(x): j=\right.$ $1, \ldots, g\}$.

Proof: See [41]. Also note that the blocks in the final design have cardinalities in $K^{\prime}$, rather than $K$.

Theorem 4.5 If $r \in R N(k)$, then a design with block size of $k$ and a group vector of $(k-1)^{r}$ exists.

Proof: Deleting a point, and using its blocks as groups in a $B(k ;(k-1) r+1)$, gives the GDD; note that this construction can be reversed; (see [25]).

\section{Specific Constructions}

In this section, we start applying the results of the previous section to produce the tools for the second of the problems, that of constructing designs whose block sizes are in $Q_{1(8)}$, where $Q_{1(8)}$ is the set of prime-powers congruent to 1 modulo 8. It will be convenient to define $U_{1(8)}$ by:

$$
t \in U_{1(8)} \Longleftrightarrow 8 t+1 \in B\left(Q_{1(8)}\right) .
$$

Lemma 5.1 There exist $\{9\}-G D D$ s of type $8^{9}$ and type $8^{10}$.

Proof: Apply Theorem 4.5 to the 73 and 81 point designs of Lemma 3.7. 
Lemma 5.2 If $q=8 t+1 \in R R N(8)$, then $56 t+8 \in R B(8)$, and $64 t+9 \in B\left(\left\{9, q^{*}\right\}\right)$, and there exists a $\{9\}-G D D$ of type $8^{7 t+1}(q-1)^{1}$, and there exists a $\left\{9, q^{*}\right\}-G D D$ of type $8^{q}$.

Proof: Complete the RBIBD with $q=8 t+1$ infinite points, and then delete either an infinite point, or a finite point, and use its lines to indicate the groups.

Theorem 5.3 If there exists a K-GDD on $v$ points, with group sizes contained in $M$, and $K \subset R N(9) \cup\left(Q_{1(8)} \backslash\{25,89\}\right)$, then $M \subset U_{1(8)}$ implies $v \in U_{1(8)}$.

Proof: Give each point a weight of 8, and apply Wilson's fundamental construction. The needed components come from Theorem 4.5 for $R N(9)$, and Lemma 3.9 via Lemma 5.2 for the other block sizes. We fill in the groups with a point at infinity to obtain the required result.

Corollary 5.4 If there exists a $\{9,10\}-G D D$ on $v$ points, with group sizes chosen from $M$, and $M \subset U_{1(8)}$, then $v \in U_{1(8)}$.

Lemma 5.5 (Code T) If $m \in T D(10)$ and $0 \leq n \leq m$ and $\{m, n\} \subset U_{1(8) \text {, then }}$ $9 m+n \in U_{1(8)}$.

Proof: Truncate one group of the transversal to size $n$, then use Corollary 5.4.

Concentrating on the points in the last block yields the "last spike" and "block deletion" constructions given below.

Lemma 5.6 (Code Ls) If $m \in T D(n)$ and $m \in U_{1(8)}$, and $n \geq 9$ and $n \in Q_{1(8)} \backslash$ $\{25,89\}$, then $9(m-1)+n \in U_{1(8)}$.

Proof: Remove $m-1$ points from $n-9$ groups of the transversal, retaining all the points of the last block, to give a $\left\{9,10, n^{*}\right\}$-GDD of type $m^{9} 1^{n-9}$. Now use Theorem 5.3.

Lemma 5.7 (Code L) If $m \in T D(n)$ and $m \in Q_{1(8)} \backslash\{25,89\}$, and $n \geq 9$ and $n \in$ $U_{1(8)}$, then $9(m-1)+n \in U_{1(8)}$.

Proof: As in Lemma 5.7, we construct a $\left\{9,10, n^{*}\right\}$-GDD of type $m^{9} 1^{n-9}$. Now fill in the groups and delete the big block to give a $\{9,10, m\}$-GDD of type $1^{9 m-9} n^{1}$, and use Theorem 5.3.

Lemma 5.8 (Code BD) If $m \in T D(10)$ and $9 \leq n \leq 10$ and $\{m, m-1\} \subset U_{1(8)}$, then $10 m-n \in U_{1(8)}$.

Proof: Remove $n$ points from one block of the transversal, then use Corollary 5.4. 
Remark 5.9 Note that if $n=10$, then $m \in U_{1(8)}$ is not needed.

Lemma 5.10 (Code R) If $v \in R B(9)$ and $8 n+1 \leq v$ and $n \in U_{1(8)}$, then $v+n \in U_{1(8)}$.

Proof: Add n new points to the blocks of $\mathrm{n}$ resolution sets, then use Corollary 5.4.

Lemma 5.11 If $m \in T D(k+1)$, then:

1. there exists a $\{k+1, m+1\}-G D D$ of type $k^{m} m^{1}$;

2. there exists a $\{k+1, m\}-G D D$ of type $k^{m}(m-1)^{1}$.

Proof: Take the transversal, and fill in groups with 1 or 0 points at infinity and then delete a finite point and use its blocks as groups to give the result.

Lemma 5.12 If $k$ is a power of 2 , then there exists a $\{k+1,2 k+1\}-G D D$ on $k(2 k+3)$ points, and this design has a group vector of $k^{2 k-1}(2 k)^{2}$.

Proof: In some ways, this lemma is a corollary of Lemma 3.2. The Seiden design can be embedded in $P G(2,2 k)$. We now consider the non-Seiden points in $P G(2,2 k)$, and delete one of these and use its blocks to define the groups.

Lemma 5.13 There exist $\{9,17\}-G D D$ s with group vectors of $8^{15} 16^{1}, 8^{17}, 8^{16} 16^{1}, 8^{17} 16^{1}$ and $8^{15} 16^{2}$.

Proof: This follows from Lemma 5.2 with $t=2$ (twice), from Lemma 5.11 with $m=16$ and $m=17$, and from Lemma 5.12 .

Mullin et al. [38, Lemma 6.12], used a construction similar to Lemma 5.14. We could also adapt their Lemma 6.20, but this yields nothing new here, as we have a rich set of constructions in the next six lemmas, covering essentially the same ranges. Five of these constructions of these lemmas were used previously by Greig and Abel [22]; Lemma 5.16 (code B) is new.

Lemma 5.14 (Code A) If $m \in T D(18)$ and $0 \leq s \leq t \leq m$ and $n=s+t$, and $\{m, 2 m, s, t\} \subset U_{1(8)}$, then $17 m+n \in U_{1(8)}$.

Proof: Truncate two groups to sizes $s$ and $t$, and give the points of these truncated groups a weight of 8 . Give the points of one other group weight 16 and give all the other points weight 8 . The needed component group vectors are $8^{15} 16^{1}, 8^{16} 16^{1}$, and $8^{17} 16^{1}$, and are obtained from Lemma 5.13, so we may apply Wilson's fundamental construction to give a design with a group vector of $(8 m)^{15}(16 m)^{1}(8 s)^{1}(8 t)^{1}$. We fill these groups in with the aid of a point at infinity. 
Remark 5.15 Note that, provided we took $s=0$, we could relax the condition $m \in$ $T D(18)$ to $m \in T D(17)$.

Lemma 5.16 (Code B) If $m$ is a prime power with $m \geq 17,0 \leq t \leq 17, n=2 t \leq$ $m+1$, and $\{m, m+2\} \subset U_{1(8)}$, then $17 m+n \in U_{1(8)}$.

Proof: Using Lemma 3.6, we may construct a $T D(17 ; m)$ with at least $t$ groups containing a pair of oval points; give these $t$ pairs of points a weight of 16 , and give all the other points a weight of 8 . The needed component group vectors are $8^{17}, 8^{16} 16^{1}$, and $8^{15} 16^{2}$, and are obtained from Lemma 5.13, so we may apply Wilson's fundamental construction to give a design with a group vector of $(8 m)^{17-t}(8(m+2))^{t}$. We fill these groups in with the aid of a point at infinity.

Lemma 5.17 (Code C) If $m \in T D(17)$ and $0 \leq s \leq t \leq m$ and $n=2 m+s+t$, and $\{m, m+s, m+t\} \subset U_{1(8)}$, then $15 m+n \in U_{1(8)}$.

Proof: Give s points from one group a weight of 16, give t points from another group a weight of 16 , and give all the other points a weight of 8 . The needed component group vectors are $8^{17}, 8^{16} 16^{1}$, and $8^{15} 16^{2}$, and are obtained from Lemma 5.13, so we may apply Wilson's fundamental construction to give a design with a group vector of $(8 m)^{15}(8 m+8 s)^{1}(8 m+8 t)^{1}$. We fill these groups in with the aid of a point at infinity.

Lemma 5.18 (Code D) If $m \in T D(17), 0 \leq s+t \leq m, n=s+2 t$, and $\{m, 2 m, n\} \subset$ $U_{1(8)}$, then $17 m+n \in U_{1(8)}$.

Proof: Give s points from one group a weight of 8 , give t points from the same group a weight of 16, give the points of another group a weight of 16, and give the points of all the other 15 groups a weight of 8 . The needed component group vectors are $8^{15} 16^{1}, 8^{16} 16^{1}$, and $8^{15} 16^{2}$, and are obtained from Lemma 5.13, so we may apply Wilson's fundamental construction to give a design with a group vector of $(8 m)^{15}(16 m)^{1}(8 s+16 t)^{1}$. We fill these groups in with the aid of a point at infinity.

Lemma 5.19 (Code E) If $m \in T D(17)$ and $1 \leq s \leq m$ and $1 \leq t \leq m$ and $15(s-1)+$ $t \leq m$ and $n=m+t$, and $\{m+1, m+s, m+t\} \subset U_{1(8)}$, then $16(m+1)+(s-1)+n \in U_{1(8)}$.

Proof: Give the points from the last block a weight of 16, give $s-1$ other points from the first group a weight of 16, give $t-1$ other points from the second group a weight of 16 , and give all the other points a weight of 8 . There are $15(s-1)$ lines containing a pair of points with weight 16, one from group 1 and the other from groups 3 through 17; when assigning the extra $t-1$ weight 16 points in the second group, we must avoid these lines; this is possible by hypothesis. The needed component group vectors are $8^{17}$, $8^{16} 16^{1}, 8^{15} 16^{2}$, and $16^{17}$, and are obtained from Lemma 5.13, or from Lemma 3.8 with $q=16$, so we may apply Wilson's fundamental construction to give a design with a group vector of $(8 m+8)^{15}(8 m+8 s)^{1}(8 m+8 t)^{1}$. We fill these groups in with the aid of a point at infinity. 
Remark 5.20 In all instances, we will take $s=1$.

Lemma 5.21 (Code F) If $m \in T D(18), 0 \leq n<m$, and $\{m-1,2 m, n\} \subset U_{1(8)}$, then $18 m-16+n \in U_{1(8)}$.

Proof: Give the points from the last block a weight of 0, except for the first group, where all points get a weight of 16 ; also give $m-n-1$ other points from the last group a weight of 0 ; and give all the other points a weight of 8 . The needed component group vectors are $8^{15} 16^{1}, 8^{16} 16^{1}$, and $8^{17} 16^{1}$, and are obtained from Lemma 5.13 , so we may apply Wilson's fundamental construction to give a design with a group vector of $(8 m-8)^{16}(16 m)^{1}(8 n)^{1}$. We fill these groups in with the aid of a point at infinity.

Remark 5.22 In all instances of the preceeding six lemmas, rather than filling the groups with the aid of a single infinite point, we could use $w>1$ infinite points, as allowed by Theorem 4.2; we shall have occassion to do this.

The Singular Indirect Product construction was introduced in [36]. The following is a particular instance.

Lemma 5.23 (Code Ir) If $56 m+8 \in R B(8)$, and $t \leq 8 m+1$, and $m+t=n \in U_{1(8)}$, and the incomplete transversal TD $(9 ; 56 m+8+t)-T D(9 ; t)$ exists, then $63 m+9+n \in$ $U_{1(8)}$.

Proof: Since $56 m+8 \in R B(8)$, we may adjoin $8 m+1$ points to the resolution sets to produce a $B\left(\left\{9,8 m+1^{*}\right\}\right)$, We use this design to fill in the groups of the incomplete transversal design with aid of $8 m+1-t$ points at infinity, with the points of the design labelled so that the single filling block of size $8 m+1$, (which is omitted), coincides with the missing subgroup and the infinite points. Filling in the infinite points and the missing subgroups with a single block of size $8(m+t)+1=8 n+1$ completes the construction.

Remark 5.24 For application in Appendix Table A.1, we will obtain the ITDs we need from Lemma 3.19, using $m=37$, or $m=107$.

\section{The Spectrum}

The objective of this section is to establish Theorem 6.1.

Theorem 6.1 If $m$ is not one of the values listed in Table 6.1, then $m \in U_{1(8)}$, and $8 m+1 \in B\left(Q_{1(8)}\right)$. 
Table 6.1.

\begin{tabular}{rrrrrrrrrr}
\multicolumn{10}{c}{ Values of $m$ for which $m \in U_{1(8)}$ is unknown. } \\
4 & 7 & 8 & 13 & 16 & 20 & 22 & 23 & 25 & 26 \\
27 & 31 & 33 & 37 & 38 & 40 & 43 & 47 & 48 & 52 \\
58 & 59 & 60 & 61 & 62 & 63 & 67 & 68 & 69 & 70 \\
76 & 79 & 85 & 88 & 89 & 92 & 93 & 94 & 98 & 103 \\
106 & 112 & 114 & 115 & 118 & 123 & 124 & 125 & 130 & 132 \\
133 & 134 & 139 & 140 & 142 & 143 & 148 & 151 & 157 & 160 \\
166 & 175 & 178 & 184 & 187 & 191 & 192 & 195 & 196 & 197 \\
199 & 202 & 203 & 204 & 205 & 208 & 209 & 211 & 213 & 214 \\
218 & 220 & 221 & 223 & 224 & 226 & 227 & 229 & 232 & 238 \\
247 & 250 & 256 & 259 & 265 & 268 & 364 & 367 & 368 & 373 \\
376 & 382 & 385 & 391 & 400 & 424 & 427 & 438 & 463 & 607 \\
623 & 628 & 637 & 643 & 646 & 670 & 823 & 832 & 1024 & 1039 \\
1048 & 1084 & & & & & & & &
\end{tabular}

We give, in appendix Table A.1, our constructions for values up to 288 together with further constructions covering portions of the range up to 1648. We begin this section by concentrating on the first 288 values, and in exhibiting subdesigns for this range.

Lemma 6.2 (Code RB) If $m \in E$, then $8 m+1 \in B\left(Q_{1(8)}\right)$ with $E$ given by: $E=$ $\{9,17,41,49,97,113,121,169,193,233,241\} \cup\{361,409,1033\}$.

Proof: This lemma is essentially a corollary of Lemma 5.2.

Lemma 6.3 (Code ZZ) If $m \in\{111,198,805,814,826\}$, then $m \in U_{1(8)}$.

Proof: For 111; take Mathon's design of Lemma 3.10, and add 12 infinite points, to give a $\{10\}$-GDD of type $3^{33} 12^{1}$; apply Lemma 5.3. For 198; take Mathon's design of Lemma 3.10, and give each point a weight of 16, and apply Wilson's Fundamental Construction of Theorem 4.4, using a $T D(9 ; 16)$. For 805 ; take a $T D(10 ; 81)$ and truncate one block to size 5; note that the short block only contains points from the full groups; fill the full groups, and remove the short block to give a $\{9,10,81\}$-GDD with group sizes from $\left\{1,80,5^{*}\right\}$, and thence a $\{9,10\}$-GDD with group sizes from $\left\{1,80,5^{*}\right\}$; the needed designs for Wilson's FC come from Lemmas 5.1. For $\{814,826\}$; truncate one group of a $T D(18 ; 23)$ to size 16 or 22 , and give each point a weight of 16 , and apply Wilson's FC; we may obtain $\{17\}$-GDDs of types $16^{17}$ and $16^{18}$ for this application by using Theorem 4.5 with $k=17$ on Lemma 3.1 with $q=16$ or 17 .

We next summarize some of the smaller designs constructions. 
Table 6.2.

\begin{tabular}{|c|c|c|c|c|}
\hline$m$ & $8 m+$ & & Authority & Remarks \\
\hline 9 & 73 & $=P G(2,8)$ & Lemma 3.1 & \\
\hline 10 & 81 & $=A G(2,9)$ & Lemma 3.1 & \\
\hline 17 & 137 & $=17+R B(8 ; 120)$ & Lemma 6.2 & \\
\hline 18 & 145 & $=9 *(17-1)+1$ & Lemma 4.3 & \\
\hline 19 & 153 & $=9 * 17$ & Lemma 4.1 & \\
\hline 28 & 225 & $=9 * 25$ & Lemma 4.1 & \\
\hline 34 & 273 & $=P G(2,16)$ & Lemma 3.1 & \\
\hline 36 & 289 & $=A G(2,17)$ & Lemma 3.1 & \\
\hline 45 & 361 & $=9 *(41-1)+1$ & Lemma 4.3 & {$[3]$ for $T D(9 ; 40)$} \\
\hline 46 & 369 & $=9 * 41$ & Lemma 4.1 & \\
\hline 53 & 425 & $=17 * 25$ & Lemma 4.1 & \\
\hline 54 & 433 & $=B(9 ; 433)$ & Lemma 3.7 & \\
\hline 55 & 441 & $=9 * 49$ & Lemma 4.1 & \\
\hline 64 & 513 & $=R B(9 ; 513)$ & Lemma 3.1 & \\
\hline 73 & 585 & $=9 *(73-9)+9$ & Lemma 4.3 & \\
\hline 74 & 593 & $=9 *(73-9)+17$ & Lemma 5.23 & $\operatorname{ITD}(9 ; 65)-(9 ; 1)$ \\
\hline 78 & 625 & $=A G(2,25)$ & Lemma 3.1 & \\
\hline 91 & 729 & $=9 * 81$ & Lemma 4.1 & \\
\hline 127 & 1017 & $=9 * 113$ & Lemma 4.1 & \\
\hline 128 & 1025 & $=25 * 41$ & Lemma 4.1 & \\
\hline 135 & 1081 & $=9 *(121-1)+1$ & Lemma 4.3 & \\
\hline 136 & 1089 & $=9 * 121$ & Lemma 4.1 & \\
\hline 137 & 1097 & $=9 *(137-17)+17$ & Lemma 4.3 & \\
\hline 138 & 1105 & $=9 *(137-17)+25$ & Lemma 5.23 & $\operatorname{ITD}(9 ; 121)-(9 ; 1)$ \\
\hline 145 & 1161 & $=9 *(137-9)+9$ & Lemma 4.3 & \\
\hline 146 & 1169 & $=9 *(145-17)+17$ & Lemma 4.3 & \\
\hline 201 & 1609 & $=25 *(73-9)+9$ & Lemma 4.3 & \\
\hline 206 & 1649 & $=17 * 97$ & Lemma 4.1 & \\
\hline 210 & 1681 & $=A G(2,41)$ & Lemma 3.1 & \\
\hline 216 & 1729 & $=9 *(193-1)+1$ & Lemma 4.3 & \\
\hline 217 & 1737 & $=9 * 193$ & Lemma 4.1 & \\
\hline 228 & 1825 & $=25 * 73$ & Lemma 4.1 & \\
\hline 240 & 1921 & $=17 * 113$ & Lemma 4.1 & \\
\hline 251 & 2009 & $=41 * 49$ & Lemma 4.1 & \\
\hline
\end{tabular}

We next turn to the range 289-3439; constructions A and C, as detailed below in Tables 6.3 and 6.4 and Remark 6.4, cover large portions of this range. In both Tables 6.3 and 6.4 , we get valid constructions for the range $17 \mathrm{~m}$ through $19 \mathrm{~m}$ with the exception of the noted failures. 
Table 6.3.

$\begin{array}{rlrrl}m & \text { Invalid } n & 17 m & 19 m & \text { Failures } \\ 17 & 33 & 289 & 323 & 322 \\ 32 & 55,63 & 544 & 608 & 599,607 \\ 41 & 79,81 & 697 & 779 & 776,778 \\ 64 & 116,122-127 & 1088 & 1216 & 1204,1210-1215 \\ 73 & 133-4,140-1 & 1241 & 1387 & 1374-5,1381-2 \\ 81 & & 1377 & 1539 & \\ 97 & 189 & 1649 & 1843 & 1838 \\ 121 & & 2057 & 2299 & \\ 127 & 250,251 & 2159 & 2413 & 2409,2410 \\ 137 & 269 & 2329 & 2603 & 2598 \\ 149 & 297 & 2533 & 2831 & 2830 \\ 163 & & 2771 & 3097 & \\ 167 & 333 & 2839 & 3173 & 3172 \\ 181 & & 3077 & 3439 & \end{array}$

Remark 6.4 Using the overlapping ranges, or applying construction C of Lemma 5.17 with the same value of $m$ to the exceptions in Table 6.3, we are able to remove all of them except 322, 607 and 1211.

Table 6.4 presents some cases of construction C. Again, apart from the noted failures, the inclusive range $17 \mathrm{~m}$ through $19 \mathrm{~m}$ has a valid construction available.

Table 6.4.

\begin{tabular}{|c|c|c|c|c|c|}
\hline$m$ & Invalid $n$ & $15 m$ & $17 m$ & $19 m$ & Failures \\
\hline 19 & $\begin{array}{l}39,41,44,46, \\
73-76\end{array}$ & 285 & 323 & 361 & $\begin{array}{l}324,326,329,331 \\
358-361\end{array}$ \\
\hline 29 & 115,116 & 435 & 493 & 551 & 550,551 \\
\hline 49 & $189,195,196$ & 735 & 833 & 931 & $924,930,931$ \\
\hline 53 & $115,116,211,212$ & 795 & 901 & 1007 & $910,911,1006,1007$ \\
\hline 71 & $280,281,283,284$ & 1065 & 1207 & 1349 & None thru 1344 \\
\hline 83 & 331,332 & 1245 & 1411 & 1577 & 1576,1577 \\
\hline 101 & $392,397,403,404$ & 1515 & 1717 & 1919 & None thru 1911 \\
\hline 107 & $409,415,421,423$, etc. & 1605 & 1819 & 2033 & None thru 2013 \\
\hline 113 & 227-8,231,443, etc. & 1695 & 1921 & 2147 & None in $1927-2137$ \\
\hline
\end{tabular}

Lemma 6.5 If $289 \leq t \leq 3439$, and $t \notin E$, then $t \in U_{1(8)}$, where $E$ is given by: $\{322,324,326,329,331\} \cup N_{358}^{492} \cup\{607\} \cup N_{609}^{696} \cup N_{780}^{832} \cup N_{1006}^{1087} \cup N_{1576}^{1648}$. 
Proof: This follows by the application of constructions A and $\mathrm{C}$ as detailed in Tables 6.3 and 6.4, noting Remark 6.4.

Most of the exception set $E$ of Lemma 6.5 can be dealt with by applying our constructions exactly as given. However, for some constructions we will use Theorem 4.2 with more than one infinite point.

Lemma 6.6 $A B\left(Q_{1(8)} \cup\left\{8 w+1^{*}\right\} ; 8(m+w)+1\right)$ exists when $(m, w)$ is one of the following pairs:

$$
\begin{array}{lllllll}
(8,1) & (16,1) & (16,2) & (17,1) & (25,3) & (27,1) & (32,2) \\
(43,6) & (50,3) & (54,1) & (81,6) & (83,1) & (89,1) &
\end{array}
$$

Proof: For $m=43$, this follows from Lemma 5.2; for other $m<81$, this follows from Table 6.2; for $m \geq 81$, this follows from Lemma 5.5, truncating a $T D(10,9)$.

We use these subdesigns in the constructions given in Table 6.5.

\section{Table 6.5.}

$\begin{array}{ccclccl}\text { Code } & m & w & \begin{array}{l}\text { Needed } \\ \text { subdesigns }\end{array} & \text { Result } & n \text { range } & \text { Successes } \\ \mathrm{T} & 16 & 1 & (16,1) & 144+n & 1 \leq n \leq 17 & \text { Several } \\ \mathrm{T} & 25 & 3 & (25,3) & 225+n & 3 \leq n \leq 28 & \text { Several } \\ \mathrm{T} & 27 & 1 & (27,1) & 243+n & 1 \leq n \leq 28 & \text { Several } \\ \mathrm{A} & 16 & 2 & (16,2)(32,2) & 272+n & 2 \leq n \leq 18 & 274,277, \\ & & & (0,2) & & & 281,283,286 \\ \mathrm{~T} & 32 & 2 & (32,2) & 288+n & 2 \leq n \leq 34 & 322 \\ \mathrm{~A} & 17 & 1 & (17,1) & 289+n & n=2 m+w=35 & 324 \\ \mathrm{~T} & 43 & 6 & (43,6) & 387+n & 6 \leq n \leq 49 & \text { Several } \\ \mathrm{D} & 25 & 3 & (25,3)(50,3) & 425+n & 3 \leq n \leq 53 & 454,457 \\ \mathrm{~A} & 27 & 1 & (27,1)(54,1) & 467+n & 1 \leq n \leq 28 & 472 \\ & & & (8,1) & & & \\ \mathrm{C} & 43 & 6 & (43,6)(81,6) & 726+n & 49 \leq n \leq 92 & 808,817 \\ \mathrm{~T} & 83 & 1 & (83,1) & 747+n & 1 \leq n \leq 84 & 831 \\ \mathrm{C} & 89 & 1 & (89,1) & 1424+n & 90 \leq n \leq 189 & 1582\end{array}$

Lemma 6.7 If $t \leq 3439$, and $t \notin T$, then $t \in U_{1(8)}$, where $T$ is the list of unconstructed cases given in Table 6.1.

Proof: Appendix Table A.1, in conjunction with Table 6.2, covers the values through 288 and most of the exception set $E$.

To deal with the values exceeding 3439, we need three preliminary lemmas.

Lemma 6.8 Any sequence of 10 consecutive integers contains at least one value from $T D(10)$. 
Proof: Consider only the 5 odd numbers of the sequence, and note that at most two are divisible by 3 , and at most one by 5 or 7 . Hence there is (at least) one value with no factors less than 11; the result then follows by Theorem 3.14.

Lemma 6.9 Let $E^{\prime}=\{367,373,391,463,607,643,823,1039\}$. If $m \notin U_{1(8)}$, and $288 \leq$ $m \leq 3439$ and $m \notin E^{\prime}$, then $m$ is divisible by 2 or 7 .

Proof: This is simply a matter of checking Table 6.1.

Lemma 6.10 $G \subset U_{1(8)} \cap T D(10)$, where $G=\{331,337,343,349,353,359,361,369$, $379,389,397,461,467,601,611,641,647,821,827,1033,1040\}$.

Proof: For $G \subset U_{1(8)}$, we check Table 6.1. For $G \subset T D(10)$, we note that all the numbers given are prime powers, except $369=9 * 41,611=13 * 47$, and $1040=16 * 64+16$.

Lemma 6.11 If $m>3439$, then $m \in U_{1(8)}$.

Proof: Note that $U_{1(8)}$ contains an unbroken sequence of values from 269 through 363 ; the range 269 through 331 contains $63>6 * 9$ values. We may use the truncated transversal construction of Lemma 5.5, with $331 \leq m \leq 361$ to construct the range $3248=9 * 331+$ 269 through 3610, with $m$ being chosen from the set $G$ of Lemma 6.10; note that the $m$ values used do not differ by more than 6 . Next, note the range 269 through 361 contains $93>10 * 9$ values. Again we use the truncated transversal construction of Lemma 5.5. By Lemma 6.8, we are assured that we may find a sequence of $m \in T D(10)$ close enough together that the ranges of the successive constructions overlap. The only problem is that some of the needed $m$ may not be in $U_{1(8)}$. The set $E^{\prime}$ of Lemma 6.9 gives the only possible problem values in the values that Lemma 6.8 is providing. These problem values can be overcome by use of values from the set $G$ of Lemma 6.10 ; the remaining values of $G$ (i.e., those exceeding 361 ), were all chosen to straddle values in $E^{\prime}$, with the straddling values differing by at most 10 .

Theorem 6.12 If $v$ is not in Table 6.6, and $v \equiv 1 \bmod 8$, then $v \in B\left(Q_{1(8)}\right)$.

Proof: This is really a restatement of Theorem 6.1 .

Table 6.6.

$\begin{array}{rrrrrrrrrr}33 & 57 & 65 & 105 & 129 & 161 & 177 & 185 & 201 & 209 \\ 217 & 249 & 265 & 297 & 305 & 321 & 345 & 377 & 385 & 417 \\ 465 & 473 & 481 & 489 & 497 & 505 & 537 & 545 & 553 & 561 \\ 609 & 633 & 681 & 705 & 713 & 737 & 745 & 753 & 785 & 825 \\ 849 & 897 & 913 & 921 & 945 & 985 & 993 & 1001 & 1041 & 1057\end{array}$


THE ELECTRONIC JOURNAL OF COMBINATORICS 7 (2000), \#R13

$\begin{array}{llllllllll}1065 & 1073 & 1113 & 1121 & 1137 & 1145 & 1185 & 1209 & 1257 & 1281 \\ 1329 & 1401 & 1425 & 1473 & 1497 & 1529 & 1537 & 1561 & 1569 & 1577 \\ 1593 & 1617 & 1625 & 1633 & 1641 & 1665 & 1673 & 1689 & 1705 & 1713 \\ 1745 & 1761 & 1769 & 1785 & 1793 & 1809 & 1817 & 1833 & 1857 & 1905 \\ 1977 & 2001 & 2049 & 2073 & 2121 & 2145 & 2913 & 2937 & 2945 & 2985 \\ 3009 & 3057 & 3081 & 3129 & 3201 & 3393 & 3417 & 3505 & 3705 & 4857 \\ 4985 & 5025 & 5097 & 5145 & 5169 & 5361 & 6585 & 6657 & 8193 & 8313 \\ 8385 & 8673 & & & & & & & & \end{array}$

\section{The Basis for $1 \bmod 8$}

Since we already have the machinery and most of the results already in place, we will take the opportunity to treat the PBD basis for the $1 \bmod 8$ case. Let $N_{1(m)}=\{v$ : $v \equiv 1 \bmod m\}$; we wish to find a set $H_{1(m)}$ containing as few elements as possible, such that $N_{1(m)}=B\left(H_{1(m)}\right)$. This problem was considered by Wilson for $2 \leq m \leq 4$, and subsequently by Mullin for $5 \leq m \leq 7$, and by Greig [41, 35, 21].

Lemma 7.1 Let a be a positive integer, and suppose that there exists a positive integer $u$ such that $u \equiv 1 \bmod a$, and that there exists both $a T D(a+1 ; u-1)$ and $a T D(a+1 ; u)$. If there exists a TD $(u+1 ; m)$, then $m(u-1)(a+1)+a t+1$ is inessential in $H_{1(a)}$ for $0 \leq t \leq m$.

Proof: See [35, Lemma 2.2]; we truncate one group of the $T D(u+1 ; m)$, and apply Wilson's FC, with group types found from the first two TDs, and then fill with an infinite point.

Let $P_{k}=\{q: q \leq k, q$ prime $\}$; we define $U\left(P_{k}\right)$ to be the smallest integer $\delta$ such that, for any positive $n$, we can always find some $s$ satisfying $n \leq s<n+\delta$ with the property that $s$ is relatively prime to every element of $P_{k}$. The main consequence of this, via MacNeish [34], is the following lemma.

Lemma 7.2 Let $k$ be a positive integer. Then given any positive integer $n$, there exists an integer $s$ such that $n \leq s<n+U\left(P_{k}\right)$ and there exists a $T D(k+2 ; s)$.

Proof: See [35, Lemma 2.1].

Let $V(a, b)=\{v: a+1 \leq v \leq b, \quad v \equiv 1 \bmod a\}$

Lemma 7.3 Let $a$ be a positive integer, and let $u$ be an integer such that $u \equiv 1 \bmod a$ and there exists a $T D(a+1 ; u-1)$ and a $T D(a+1 ; u)$. Let $\delta=U\left(P_{k}\right)$ and let $w$ be an integer such that there exists a $T D(u+1 ; w)$ and $w \geq \delta(u-1)(a+1) / a-1$. Then the set $V(a, w(u-1)(a+1)-a+1)$ is a finite basis for $N_{1(a)}$. 
Proof: See [35, Theorem 2.3]; we give a slightly improved version, with the improvement resulting from the observation that consecutive intervals need only abut each other, not overlap; the hypothesized bound on $w$ is decreased by unity.

Corollary $7.4 V(8,6401)$ is a basis for $N_{1(8)}$.

Proof: Take $a=8, u=9,10=\delta=U\left(P_{9}\right)=U\left(P_{7}\right)$, and $w=89$. It follows from Lemma 6.8 that we can take $\delta=10$.

Actually, since the prime powers below 89 are within 6 of each other, there is more overlap of consecutive intervals than is provided for in Lemma 7.3, with its allowed gap of 10 , so the result of Corollary 7.4 can be improved on considerably.

Lemma 7.5 Let $S=N_{1}^{80} \cup N_{91}^{98} \cup N_{111}^{116} \cup N_{131}^{143} \cup N_{191}^{206}$. If $m \notin S \cup N_{321}^{332}$, then $8 m+1$ is inessential in $H_{1(8)}$.

Proof: In Lemma 7.1, take $a=8, u=9$, and let $m$ range through the prime powers from 9 through 83.

Lemma 7.6 If $m \in N_{321}^{332}$, then $8 m+1$ is inessential in $H_{1(8)}$.

Proof: In Lemma 5.14, take $m=17$ or $m=19$.

Lemma 7.7 If $E=\{9,17,41,49,97,113,193\} \cup\{33,57,65\}$, then $8 q+1$, with $q \in E$, is inessential in $H_{1(8)}$.

Proof: This lemma is essentially a corollary of Lemma 5.2, and Lemma 3.8.

Lemma 7.8 We have $125 \in B\left(\left\{5^{*}, 9,11,13\right\}\right)$, and also the incomplete transversal design $T D(9 ; 125)-T D(9 ; 5)$ exists.

Proof: The PBD was constructed in [21] from an $R T D(10 ; 13)$ in $P G(2,13)$ and parallels the construction of the 158 point PBD in Lemma 8.2. The ITD result follows from this PBD.

Table 7.1.

$\begin{array}{rrlll}m & 8 m+1 & & \text { Authority } & \text { Remarks } \\ 63 & 505 & =9 *(57-1)+1 & \text { Lemma } 4.3 & K=\{9,57\} \\ 68 & 545 & =17 *(33-1)+1 & \text { Lemma } 4.3 & K=\{17,33\} \\ 72 & 577 & =9 *(65-1)+1 & \text { Lemma } 4.3 & K=\{9,65\} \\ 132 & 1057 & =P G(2,32) & \text { Lemma } 3.1 & K=\{33\} \\ 142 & 1137 & =9 *(137-17)+57 & \text { Lemma } 5.23 & K=\{9,57\} \\ & & & \text { Lemma } 7.8 & \operatorname{ITD}(9 ; 125)-(9 ; 5) \\ 199 & 1593 & =9 * 177 & \text { Lemma } 4.1 & K=\{9,177\} \\ 200 & 1601 & =25 *(65-1)+1 & \text { Lemma } 4.3 & K=\{25,65\} \\ 202 & 1617 & =33 * 49 & \text { Lemma } 4.1 & K=\{33,49\}\end{array}$


THE ELECTRONIC JOURNAL OF COMBINATORICS 7 (2000), \#R13

Theorem 7.9 If $m$ is not listed in Table 7.2, then $8 m+1$ is inessential in $H_{1(8)}$.

Proof: We only have to deal with elements of $S$ from Lemma 7.5. We use Corollary 3.4 to remove $m \in\{19,37,66,70\}$; we use Lemma 7.7 to remove $m \in\{9,17,33,41,49,57$, $65,97,113,193\}$; we use Lemma 3.7 to remove $m \in\{10,54,73\}$; we use Lemma 6.3 to remove $m \in\{111,198\}$; the remaining constructions are to be found in Table 6.2 , or Table 7.1.

Table 7.2.

Values of $m$ for which $8 m+1 \in B\left(H_{1(8)} \backslash\{8 m+1\}\right)$ is unknown.

$\begin{array}{rrrrrrrrrr}1 & 2 & 3 & 4 & 5 & 6 & 7 & 8 & 11 & 12 \\ 13 & 14 & 15 & 16 & 20 & 21 & 22 & 23 & 24 & 25 \\ 26 & 27 & 29 & 30 & 31 & 32 & 35 & 38 & 39 & 40 \\ 42 & 43 & 44 & 47 & 48 & 50 & 51 & 52 & 56 & 58 \\ 59 & 60 & 61 & 62 & 67 & 69 & 71 & 75 & 76 & 77 \\ 79 & 80 & 92 & 93 & 94 & 95 & 96 & 98 & 112 & 114 \\ 115 & 116 & 131 & 133 & 134 & 139 & 140 & 141 & 143 & 191 \\ 192 & 194 & 195 & 196 & 197 & 203 & 204 & 205 & & \end{array}$

Theorem 7.10 If $v$ is not listed in Table 7.3, then $v$ is inessential in $H_{1(8)}$.

Proof: This is just another version of Theorem 7.9. The first 14 values are essential.

Table 7.3.

Values of $v$ for which $v \in B\left(H_{1(8)} \backslash\{v\}\right)$ is unknown.

$\begin{array}{rrrrrrrrrr}9 & 17 & 25 & 33 & 41 & 49 & 57 & 65 & 89 & 97 \\ 105 & 113 & 121 & 129 & 161 & 169 & 177 & 185 & 193 & 201 \\ 209 & 217 & 233 & 241 & 249 & 257 & 281 & 305 & 313 & 321 \\ 337 & 345 & 353 & 377 & 385 & 401 & 409 & 417 & 449 & 465 \\ 473 & 481 & 489 & 497 & 537 & 553 & 569 & 601 & 609 & 617 \\ 633 & 641 & 737 & 745 & 753 & 761 & 769 & 785 & 897 & 913 \\ 921 & 929 & 1049 & 1065 & 1073 & 1113 & 1121 & 1129 & 1145 & 1529 \\ 1537 & 1553 & 1561 & 1569 & 1577 & 1625 & 1633 & 1641 & & \end{array}$

\section{Prime Powers Exceeding Seven}

The main aim of this section is to improve the spectrum of $B\left(Q_{\geq 8}\right)$, where $Q_{\geq 8}$ is the set of all prime powers exceeding 7 . This problem was introduced by Bennett [9] and further discussed in [13]. Direct constructions for the values 123, 156, 158, 254, 316, and 1262 are given in [21], and for 291 and 295 were given in Corollary 3.5. The 123 point design takes the 120 point Denniston arc used in Corollary 3.4.1 and adds three extra 
points on an 8-line. The 158 point design uses the $q-x$ construction from [21] (with $q=16$ and $x=6)$ : start with $P G(2, q)$, and remove a point and $x+1$ lines through it to produce a $R T D(q-x ; q)$ and then take a $(q-x)$-line (in the plane) and flip the status of its $q+1$ points, now deleting $q-x$ and restoring $x+1$, and finally add a non-planar point to the $(x+1)$-line and the $q-x(q-1)$-groups. The remaining constructions are based on Brouwer [16], who considers the cyclic difference set for $P G(2, q)$, and looks at the points belonging to a residue set modulo $w$, for $w$ a divisor of $q^{2}+q+1$. Here however, we take more than one residue set; we take twelve modulo 21 sets in $P G(2,16)$, two modulo 3 sets in $P G(2,19)$, four modulo 7 sets in $P G(2,23)$, or two modulo 3 sets in $P G(2,43)$ to get our designs; if $w=3$, it doesn't matter which sets we pick; in the other two cases we used the eleven used sets plus 0 , and the four least used sets, considering the use in the difference set given by Baumert [8].

Theorem $8.1\{123,291,295,316,574,814,1262\} \subset B\left(Q_{\geq 8}\right)$.

Proof: See [21] or Corollary 3.4, or apply the $q-x$ construction with $q=32$ and $x=14$ or 6 .

We high-light two particularly useful values:

Lemma $8.2156 \in B(\{8,9,11,17\})$ and $158 \in B\left(\left\{8^{*}, 9,11,16\right\}\right)$.

Corollary 8.3 $158 \in T D(9)$.

Lemma 8.4 1. If $T D(k ; m)$ exists, then $k m \in B(\{k, m\})$, and all block sizes occur.

2. If $T D(k+1 ; m)$ exists, and $0<t<m$, then $k m+t \in B(\{k, k+1, m, t\})$, and all block sizes occur.

Proof: See Bennett [9, Lemma 2.8].

Lemma 8.5 If $T D(t ; m)$ exists, and $k+1 \leq t$, then $k m+t-k \in B(\{k, k+1, m, t\})$, and all block sizes occur.

Proof: See Bennett [9, Lemma 2.11].

Using the notation $T D^{*}(k ; m)$ to denote a $T D(k ; m)$ with at least one parallel class of $k$-lines, we have the following lemma which was exploited by Bennett and Zhu [13].

Lemma 8.6 If a $T D(k ; p)$ exists, and a $T D^{*}(p ; q)$ exists, and a $T D^{*}(k ; q)$ exists, then $a\{k, p, q\}-G D D$ of type $(k p)^{q}$ exists.

Proof: Filling in the groups of the third TD, and removing a parallel class produces a $\{k, q\}$-GDD of type $k^{q}$. Now a similar construction with the second TD shows we have a $\{p, q\}$-GDD of type $p^{q}$. Applying Wilson's FC with weight of $p$ for all points in our first constructed design, using the second constructed design to give the ingredients for the blocks of size $q$, and using the first hypothesized design to give the ingredients for the blocks of size $k$, shows the required result. 
Lemma 8.7 If $m \neq 4$, then $8 m+1 \in B\left(Q_{\geq 8}\right)$.

Proof: See Bennett [9, Theorem 4.1].

Lemma 8.8 1. If $m \notin\{3,5,6,7\}$, then $8 m \in B\left(Q_{\geq 8}\right)$;

2. If $m \geq 8$ and $m \neq 22$, then $8 m \in B\left(Q_{\geq 8} \cup\left\{8^{*}\right\}\right)$;

Proof: For the first part, see Bennett [9, Theorem 4.2]. Actually a careful analysis of Bennett's proof establishes most of the second part. It $n \geq 8$ is odd and $n \notin E_{9}$, where $E_{9}$ is a set of 30 numbers, then a $T D(9 ; n)$ is known to exist, and Bennett also shows that $n \in B\left(Q_{\geq 8}\right),\left[9\right.$, Theorem 4.1]. Hence, if $n \notin E_{9}$, then we have the result for $m=n$ by Lemma 8.4.1, and for $m=n+1$ by Lemma 8.4.2 with $t=8$. Lemma 8.4.1 also deals with the case that $m \geq 8$ is a power of 2 . Lemma 8.4.2 with $t=16,32$ also gives the cases $m=n+2$ and $m=n+4$ if $n$ is not too small. Lemma 8.5 with $t=32$ gives the cases $m=n+3$ for $n \geq 31$, and with $t=72$ gives $m=78$. We can use Lemma 8.4.2 with $k=16, t=8$ to deal with $m=35,95$. We also note that an $R B(8 ; v)$ exists for $v=120,288,680$; (the latter deals with $m=94$ ). This abbreviated proof establishes the second part of the lemma; Bennett's result now follows by noting that the missing smaller $m$ are all powers of 2 , and $8 * 22=11 * 16$, and we can again apply Lemma 8.4.1.

\section{Lemma 8.9 (Bennett $[10]$ )}

1. If $n=10$ or $12 \leq n \leq 19$, then $8 n+12 \in B\left(Q_{\geq 8} \cup\left\{w^{*}\right\}\right)$ for $8 \leq w \leq 12$;

2. If $n=12$ or $14 \leq n \leq 19$, then $8 n+14 \in B\left(Q_{\geq 8} \cup\left\{w^{*}\right\}\right)$ for $8 \leq w \leq 14$;

3. If $n \in\{11,13,19\}$ or $15 \leq n \leq 17$, then $8 n+w \in B\left(Q_{\geq 8} \cup\left\{w^{*}\right\}\right)$ for $w \leq 11$.

Proof: If $w \leq n$, we can truncate one group of a $T D(9 ; n)$ to size $w$ whenever $n$ is a prime power, and if $n=15$, we can add $w$ points to a $R B(8 ; 120)$. If $w \leq n+2$ and $n+1$ is a prime power, we can apply Lemma 8.5 to a $T D(w ; n+1)$. Finally, for $n=14$, the $R B(8 ; 120)$ is embedded as a $\{120 ;\{0,8\}\}$-arc in $P G(2,16)$; we can extend an 8 -secant to size $w$.

\section{Lemma 8.10 (Bennett [10])}

1. If $m=136$ or $138 \leq m \leq 146$ or $m \in\{148,149\}$, then $8 m+156 \in B\left(Q_{\geq 8}\right)$;

2. If $m=138$ or $140 \leq m \leq 146$ or $m \in\{148,149\}$, then $8 m+158 \in B\left(Q_{\geq 8}\right)$; 
Proof: Let $u \in\{156,158\}$; for $m \leq 144$, set $w=u-144$ and truncate one group of a $T D(9 ; 144)$; now add $w$ infinite points, and fill the groups using Lemma 8.9 for all but the last group (on $144+w$ points) which we fill with a $B\left(Q_{\geq 8} ; u\right)$ design from Lemma 8.2. For $m=145$, set $w=u-144$ and remove 8 collinear points from a $T D(9 ; 19)$, and use this as the master design in Wilson's Fundamental Construction of Theorem 4.4, giving all points weight 8 , to get a $\{8,9\}$-GDD of type $144^{8} 152^{1}$; now add $w$ infinite points, and fill the groups using Lemma 8.9 for all but the last group (on $144+w$ points) which we fill with a $B\left(Q_{\geq 8} ; u\right)$ design from Lemma 8.2. For $m>145$, set $w=u-152$ and truncate one group of a $T D(9 ; 152)$; now add $w$ infinite points, and fill the groups using Lemma 8.9 for all but the last group (on $152+w$ points) which we fill with a $B\left(Q_{\geq 8} ; u\right)$ design from Lemma 8.2.

Lemma 8.11 If $T D(k ; v-u)$ exists, and $v \in B\left(Q_{\geq 8} \cup\{u\}^{*}\right)$, and $v \in B\left(Q_{\geq 8}\right)$, then $k(v-u)+u \in B\left(Q_{\geq 8}\right)$.

Proof: See Bennett [9, Lemma 2.12].

Theorem 8.12 Let $0 \leq y \leq u-x$ and $0 \leq a-y \leq v-(u-x)$ and suppose:

1. $\{k, k+1\} \subset B\left(Q_{\geq 8}\right)$,

2. the incomplete transversal $T D(k+1 ; v)-T D(k+1 ; a)$ exists,

3. $v+x \in B\left(Q_{\geq 8} \cup\{a+x\}^{*}\right)$.

Further suppose either $(A)$ :

4. $u \in B\left(Q_{\geq 8}\right)$,

5. $k a+x+y \in B\left(Q_{\geq 8} \cup\{x+y\}^{*}\right)$;

or else $(B)$ :

$4^{\prime} . u \in B\left(Q_{\geq 8} \cup\{x+y\}^{*}\right)$,

$5^{\prime} . k a+x+y \in B\left(Q_{\geq 8}\right)$;

then $k v+u \in B\left(Q_{\geq 8}\right)$.

Proof: This is a variation of the usual singular indirect product construction. We add $x$ infinite points to the design, then truncate one of the groups of the ITD, leaving $u-x$ points with $y$ of them in the missing part. The initial conditions on $x$ and $y$ ensure that we actually remove a non-negative number from both the missing and non-missing parts of the groups of the ITD, and also leave a non-negative number of points in each part of the group. We then use $D_{3}$ to fill the $k$ untruncated groups, omitting the flat which we align with the infinite points and the hole. For version B, we then use $D_{4^{\prime}}$ to fill the last, truncated, group, and finally use $D_{5^{\prime}}$ to fill the holes and infinite points. For version A, we reverse this order, and use $D_{5}$ first, then $D_{4}$. 
Corollary 8.13 If $160 \leq m \leq 170$, or $192 \leq m \leq 279$, and $u \in\{156,158\}$, then $8 m+u \in B\left(Q_{\geq 8}\right)$.

Proof: We will apply version A of Theorem 8.12, with $k=8$ and $x=0$. Express $m$ as $m=v=8 q+a$ with $q$ taken from 19, 23, 25, 27, 29, 31, and with $8 \leq a \leq q$; this form provides us with the ITD, and also with $D_{3}$ since $x=0$. Take $y=8$ for $q=19$ or $a=22$, and $y=0$ otherwise. Lemma 8.2 gives us $D_{4}$; and since $k a+x+y$ is a multiple of 8 , Lemma 8.8 gives us $D_{5}$ for both values of $y$, noting in the case $y=0$ the subdesign is trivial.

Corollary 8.14 Let $144 \leq m \leq 159$, or $180 \leq m \leq 187$.

1. If $m \neq 146$, then $8 m+156 \in B\left(Q_{\geq 8}\right)$.

2. If $m \notin\{145,147,148,149\}$, then $8 m+158 \in B\left(Q_{\geq 8}\right)$.

Proof: We will apply version B of Theorem 8.12, with $k=8$. For $144 \leq m \leq 159$, let $m=v=144+a$ and $y=a$; the ITD follows from Wilson's MOLS construction [40] and $v=16 * 9+a$ for $a \leq 9$, or from $v=8 * 19+(a-8)$ and for $8<a \leq 15$. For $u=156$, let $x=17-a$, and for $u=158$, let $x=16-a ; D_{3}$ follows from $v+x=8 * 19+(x+a-8)$ and Lemma 8.5, and $D_{4^{\prime}}$ follows from Lemma 8.2, and $D_{5^{\prime}}$ is on $8 a+17$ or $8 a+16$ points, which is alright by Lemma 8.7, unless $a=2$ for $u=156$, or else is alright by Lemma 8.8, unless $a \in\{1,3,4,5\}$ for $u=158$.

For $u \in\{156,158\}$, if $180 \leq m \leq 187$, let $m=v=176+a$, let $x=11-a$, and $y=a-2$; the ITD follows from $v=16 * 11+a$, and $D_{3}$ follows from $v+x=11 * 17$ and Lemma 8.4.1, and $D_{4^{\prime}}$ follows from Lemma 8.2, and $D_{5^{\prime}}$ is on $8 a+9$ points, which is alright, by Lemma 8.7, for $4 \leq a \leq 11$.

Lemma 8.15 If $171 \leq m \leq 191$, and $m \notin\{174,175,188\}$, then $8 m+u \in B\left(Q_{\geq 8}\right)$ for $u \in\{156,158\}$.

Proof: For the odd values not covered by Corollary 8.14, and for 176, we may apply Lemma 8.4.2 with $t=u$ and $k=8$. For the remaining $m$, we note that $m+1$ is a prime, and apply Lemma 8.5 with $t=u$ and $k=8$.

Lemma 8.16 If a $T D(14 ; m)$ exists, $0 \leq s, t, w, x, y, z \leq m$ and $x+y+z \leq m$, then there is a $Q_{\geq 8}-G D D$ of type $(8 m)^{10}(8 x+9 y+10 z)^{1}(8 s)^{1}(8 t)^{1}(8 w)^{1}$.

Proof: See Bennett [9, Lemma 3.11].

Corollary $8.171558 \in B\left(Q_{\geq 8}\right)$

Proof: Apply Lemma 8.16 with $m=17, s=x=1, t=4, z=15$ and $w=y=0$ to give a GDD of type $136^{10} 158^{1} 8^{1} 32^{1}$. 
Theorem 8.18 If $138 \leq m \leq 279$, then $8 m+156 \in B\left(Q_{\geq 8}\right)$.

Proof: Combining the preceeding corollaries and lemmas, we only have to deal with the three exceptions of Lemma 8.15, and these are covered by Lemma 8.4 with $\mathrm{k}=31$, using the prime powers 49 and 53 .

Theorem 8.19 If $140 \leq m \leq 279$, then $8 m+158 \in B\left(Q_{\geq 8}\right)$.

Proof: The proof is essentially the same as for Theorem 8.18. The use of Lemma 8.4 with $k=31$ and the prime powers $43,49,53$, resolves $m=147$, and two of the three exceptions of Lemma 8.15, and Corollary 8.17 resolves the third exception.

We turn now to the construction of some miscellaneous values. In addition to the values mentioned in the beginning of this section, we also have:

Theorem $8.201102 \in B\left(Q_{\geq 8}\right)$.

Proof: Apply Lemma 8.6 with $k=9, p=q=11$ to give a $\{9,11\}$-GDD of type $99^{11}$, and fill with 13 infinite points using a $B(\{8,9,13\} ; 112=99+13)$ provided by $112=8 * 13+8$.

Remark 8.21 A construction similar to this one was used by Bennett and Zhu to remove this and two other values in [13]. In the introduction to this section, we mentioned the construction of adding $t-8$ extra points from an 8 -line through a 120 point Denniston arc in $P G(2,16)$; if we use the lines through a further point on this line to generate the groups, we have a $\{8,9\}$-GDD of type $8^{14} t^{1}$ for $8 \leq t \leq 16$ (and in particular $t=15$ ); taking this in conjunction with the method used in the proof of [9, Lemma 3.9] and with $254 \in B\left(Q_{\geq 8}\right)$ accounts for the other constructions in [13]. Actually, This particular GDD, in conjunction with Bennett [9, Lemma 3.8], enables us to extend Lemma 8.16 to allow six further groups receiving weights of some mix of 0 and 8 , if we wish, Provided, of course we were weighting a $T D(20 ; m))$.

Theorem 8.22 $\{1182,1220,1222,1244,1260,1268,1946\} \subset B\left(Q_{\geq 8}\right)$.

Proof: For 1182, use Brouwer's stacking Baer sub-planes construction of [16] to give 7 $P G(2,4)$ s in $P G(2,16)$, i.e., a $B(\{7,11\} ; 147)$, and to this add 6 infinite points for the resolutions on the short blocks to give a $B\left(\left\{8,11,6^{*}\right\} ; 153\right)$; use this design to fill 7 groups of a $T D(8 ; 147)$ with 6 infinite points, and then a $B(\{9,17\} ; 153)$ from $153=9 * 17$ to fill the last group. For the next two values, let $u \in\{156,158\}$ and let $a=u-152$; now use $u=8 * 19+a \in B\left(\left\{8,9,19, a^{*}\right\}\right)$ to fill in all but the last group of a $T D(8 ; 152)$ with $a$ added infinite points, and use Lemma 8.2 for the last group. The next two values are the two smallest values given by Lemma 8.10. For the last two values, we use Lemma 8.11, noting that $1268=9 *(156-17)+17$ and $1946=13 *(158-9)+9$. 
Remark 8.23 For the applications discussed in $[9,13]$, the following equations are worth noting:

1. $1578=11 *(158-16)+16$, and

2. $1626=11 *(156-9)+9$.

We may summarize the main results of this section:

Theorem 8.24 If $v$ has the value 4 or 6 modulo 8 , and $v \geq 1276$, then $v \in B\left(Q_{\geq 8}\right)$.

Proof: Given in Theorems 8.18 and 8.19.

Remark 8.25 We have now substantiated the majority of the improvements made in [12, Table 3.18] over the results in [9]. We have no construction for 1578 or 1626, the two values noted in Remark 8.23; it would appear that these were erroneously omitted from [12, Table 3.18]. In addition, we have noted constructions for the values 316, 574, 814, 1244, 1260 and 1318 which further improve [12, Table 3.18].

\section{Update}

In this final section, we wish to provide some updates and corrections to the three tables of generating/exception sets given in [12, Tables III.3.17-19].

\subsection{Table III.3.17}

Most of the changes here involve designs with $4 \in K$, and result from improvements to [37] during its later drafts. That paper has a couple of weak entries in the summary table [37, pp. 74-75]: $(55 \in B(\{4,6,8,9\}), 30 \notin B(\{4,7,8,9\}))$. It is known that $44,45,47 \notin B(\{4,7,8\})$ and also that $48 \notin B(\{4,7,9\})$. The remaining improvements with $4 \in K$ from [37] are in constructing designs; another results from Ling [29, p. 57] who gives a $B(\{4,5\} ; 104)$ with 21 parallel classes of 4 -blocks, so adding 11 points gives a $B\left(\left\{4,5,11^{*}\right\} ; 115\right)$. For $4 \in K$, we have chosen to list the current possible exceptions for all such listed $K$, several of which seem close to solution. 


\begin{tabular}{cl}
$K$ & Possible exceptions \\
\hline 4,6 & $33,34,39,45,46,51,75,87$ \\
4,8 & $48,53,60,65,69,77,89,101,161,164,173$ \\
4,9 & $60,69,84,93,96,192$ \\
$4,5,7$ & $30,39,42,51,54$ \\
$4,5,11$ & $38,42,43,46,50,54,58,62,66,67,70,74$ \\
& $78,82,90,94,98,102,106,114,118,126$ \\
$4,6,7$ & $33,45,87$ \\
$4,6,8$ & $33,34,35,39,41,47,50,51,53,59,62,65$, \\
& $71,75,77,87,89,95,101,110,131,161,170$ \\
$4,6,9$ & $34,75,87$ \\
$4,7,8$ & $48,51,54,59,62,65,66,69,74,75,77,78,83,87$, \\
& $89,90,101,102,110,111,114,123,126,131,135,143$, \\
$4,7,9$ & $150,159,161,162,164,167,170,173,174,186,195$ \\
$4,8,9$ & $111,114,138,147,150,159,174,183,186,195,210$ \\
$4,5,7,8$ & $38,53,60,69,77,101,161,164,173$ \\
$4,5,7,9$ & $30,51,54$ \\
$4,5,7,11$ & $30,42,54$ \\
$4,6,7,8$ & $33,35,41,65,77,131$ \\
$4,6,8,9$ & $34,35,41,47,50,53,59,62,71$, \\
& $75,77,87,95,101,131,161,170$ \\
$4,7,8,9$ & $35,38,39,41,42,44,47,48,51,54,59,62$, \\
& $110,143,150,159,161,164,167,173,174$ \\
$4,5,7,8,9$ & 30,51 \\
$4,6,7,8,9$ & 35,41 \\
$4,5,7,9,10,11$ & 30 \\
$4,5,9,11,19,31$ & $38,42,43,46,50,54,62,66,74,78,90,98,102,114,126$ \\
\hline
\end{tabular}

There were three changes involving designs with $5 \in K$ resulting from improvements to [11] during its final draft. The remaining improvements to [12, Table III.3.17] come from other results in the table or from three designs: Bierbrauer [14] exhibits a pentario in $P G(2,8)$ (i.e., a set of 3 quintuples, any pair of which form a hyperoval), and the pentario forms a $\{15 ;\{0,2,3\}\}$-arc in the plane, so deleting the pentario and an external line gives a $B(\{5,6,8\} ; 49)$; there are over $10^{6}$ such configurations, and using the Singer difference set given by powers of 2 in $Z_{73}$, an example set of deleted points is:

$$
\{1,2,11,27,60\}\{0,9,29,45,58\}\{7,30,38,46,71\}\{3,4,6,10,18,34,39,57,66\}
$$

The two other designs are 7-GDDs of types $7^{25}$ and $7^{37}$ constructed by Janko and Tonchev [27] and by Abel [2], and modifying these designs give the remaining new PBDs.

\begin{tabular}{cl}
$K$ & Change \\
\hline 5,7 & $259,709 \in B(K)$ \\
$5,6,8$ & $49,307 \in B(K)$ \\
$5,6,8,9$ & $174 \in B(K)$ \\
$5,7,8,9$ & $163,243 \in B(K)$ \\
$6,7,8$ & $170,174,175,176 \in B(K)$ \\
$6,7,8,9$ & $137,170,174,175,176 \in B(K)$ \\
$7,8,9$ & $175,176,259,260 \in B(K)$ \\
\hline
\end{tabular}


Finally, we note that $[29$, p. 21] contains an arithmetic error; this affects part of [30, Lemma 4.7], for $(94,95)$. More specifically, Ling gives a (lower) bound up to which we safely can add points to a certain configuration in a projective plane, although he mis-evaluated the bound here; using essentially Ling's plan, one can exceed this bound with carefully chosen points, and this was demonstrated in [7, Lemma 4.18], correcting the lacuna.

\subsection{Table III.3.18}

First, we note the errors in this table. For $K=Q_{\geq 4}$ clearly the prime powers $11,19,23$, 27 are not exceptions, nor is 51 a possible exception (truncate one group of a $T D(5 ; 11$ ) to size 7); incidentally, the exception set can be deduced from the $K=\{4,5,7,8,9\}$ exception set. Earlier, we commented on the other possible errors in this table for $K=$ $Q_{\geq 8}$ in Remark 8.25, (1578, 1626 omitted), as well as the improvements of constructions for 316, 574, 814, 1244, 1260, 1318; Bennett [10] has also obtained constructions for 1196, 1198, 1212, 1214, 1236, 1238, 1246, 1254, 1270, 1706, 1770, 1778, 1794 and all values $\geq 1810$.

In [6], it is shown that:

$$
\{161,231,266,276,296,376,561,741,946\} \subset B\left(Q_{1 \bmod 5} \cup\{6\}\right) .
$$

The Janko-Tonchev and Abel designs [27, 2] also show

$$
\{175,176,259,260\} \subset B\left(Q_{0,1 \bmod 7}\right) .
$$

\subsection{Table III.3.19}

There is one error: clearly 15 is an essential element of $H_{0,1(5)}$.

In [6], for $H_{1(5)}$, it is shown that the following are inessential:

$\{131,146,166,196,221,226,231,251,261,266,296,316,326,351,356\}$.

\subsection{Updated References}

Finally we note that the references in $[37,11]$ are more accurate than those in $[12$, Table III.3.19], and give the following updates to the reference section of [12]. 


\begin{tabular}{cc} 
Citation in $[12]$ & Citation in this paper \\
\hline$[4]$ & {$[11]$} \\
{$[12]$} & This paper itself \\
{$[13]$} & {$[21]$} \\
{$[14]$} & {$[24]$} \\
{$[15]$} & {$[23]$} \\
{$[19]$} & {$[30]$} \\
{$[20]$} & {$[31]$} \\
{$[21]$} & {$[32]$} \\
{$[25]$} & {$[37]$} \\
\hline
\end{tabular}

\section{Acknowledgement}

I would like to acknowledge the help and advice of Frank Bennett, and his hospitality during a visit to Mount Saint Vincent University, where some of this research was done. I would also like to thank Frank for his help in providing some of the constructions that went into generating [12, Table 3.18], and to Julian Abel, who also provided help with the update section.

The problem of Section 8 was one of the first combinatorial problems I tackled, and in these last ten years, I have had discussions and preprints which have helped me with the problems in this paper, and (especially for this paper) help with references, and for all of this, I would like to thank Julian Abel, Frank Bennett, Juergen Bierbrauer, Charlie Colbourn, Dieter Gronau, Martin Gruettmueller, Alan Ling, Ron Mullin, Paul Schellenberg and Vladimir Tonchev.

\section{References}

[1] R. J. R. Abel, Difference families, in: CRC Handbook of Combinatorial Designs, (C. J. Colbourn and J. H. Dinitz, eds.), CRC Press, Boca Raton FL, (1996), 270287.

[2] R. J. R. Abel, Some new BIBDs with block size 7. J. Combin. Des. (to appear).

[3] R. J. R. Abel, A.E. Brouwer, C. J. Colbourn and J.H. Dinitz, Mutually Orthogonal Latin Squares (MOLS), in: CRC Handbook of Combinatorial Designs, (C. J. Colbourn and J. H. Dinitz, eds.), CRC Press, Boca Raton FL, (1996), 111-142.

[4] R. J. R. Abel, C. J. Colbourn and J. H. Dinitz, Incomplete MOLS, in: The CRC Handbook of Combinatorial Designs, (C. J. Colbourn and J. H. Dinitz, eds.), CRC Press, Boca Raton FL, (1996), 142-172.

[5] R. J. R. Abel, N. J. Finizio, M. Greig and S. J. Lewis, Pitch tournament designs and other BIBDs - existence results for the case $v=8 n+1$, (1999), preprint; (Congr. Numer., to appear). 
THE ELECTRONIC JOURNAL OF COMBINATORICS 7 (2000), \#R13

[6] R. J. R. Abel and M. Greig, Some new $(v, 5,1)$ RBIBDs and PBDs with block sizes $\equiv 1 \bmod 5$, Australasian J. Combinat. 15 (1997), 177-202.

[7] R. J. R. Abel, M. Greig, Y. Miao and L. Zhu, Resolvable BIBDs with block size 7 and index 6, (preprint).

[8] L. D. Baumert, Cyclic Difference Sets, Lecture Notes in Math. 182 (1971).

[9] F. E. Bennett, Pairwise balanced designs with prime power block sizes exceeding 7 , Ann. Discrete Math. 34 (1987), 43-64.

[10] F. E. Bennett, Personal communications, (Oct./Nov., 1999).

[11] F.E. Bennett, C. J. Colbourn and R. C. Mullin, Quintessential pairwise balanced designs, J. Statist. Plann. Inference 72 (1998), 15-66.

[12] F. E. Bennett, H.-D. O. F. Gronau, A. C. H. Ling and R. C. Mullin, PBD-Closure, in: The CRC Handbook of Combinatorial Designs, (C. J. Colbourn and J. H. Dinitz, eds.), CRC Press, Boca Raton FL, (1996), 203-213.

[13] F. E. Bennett and L. Zhu, Conjugate-orthogonal latin squares and related structures, in: Contemporary Design Theory: A Collection of Surveys, (J. H. Dinitz and D. R. Stinson, eds.), John Wiley, New York, (1992), 41-96.

[14] J. Bierbrauer, The maximal size of a 3 -arc in $P G(2,8)$, (preprint).

[15] R. C. Bose, On the application of finite projective geometry for deriving a certain series of balanced Kirkman arrangements, Cal. Math. Soc. Golden Jubilee Commemoration Volume, Part II (1958-1959), 341-356.

[16] A. E. Brouwer, A series of seperable designs with application to pairwise orthogonal latin squares, European J. Combin. 1 (1980), 39-41.

[17] M. Buratti, A powerful method of constructing difference families and optical orthogonal codes, Des. Codes Cryptogr. 5 (1995), 13-25.

[18] C. J. Colbourn and J. H. Dinitz, Home page for The CRC Handbook of Combinatorial Designs,

URL: http://www.emba.uvm.edu/ dinitz/hcd.html

[19] R. H. F. Denniston, Some maximal arcs in finite projective planes, J. Combin. Theory 6 (1969), 317-319.

[20] M. Greig, Some group divisible design constructions, J. Combin. Math. Combin. Comput. 27 (1998), 33-52.

[21] M. Greig, Designs from projective planes, and PBD bases, J. Combin. Des. 7 (1999), $341-374$. 
THE ELECTRONIC JOURNAL OF COMBINATORICS 7 (2000), \#R13

[22] M. Greig and R. J. R. Abel, Resolvable balanced incomplete block designs with a block size of 8, Des. Codes Cryptogr. 11 (1997), 123-140.

[23] H.-D. O. F. Gronau, V. Leck, R. C. Mullin and P. J. Schellenberg, On orthogonal covers of $\vec{K}_{n}$, (preprint).

[24] H.-D. O. F. Gronau, R. C. Mullin and C. Pietsch, The closure of all subsets of $\{3,4, \ldots, 10\}$ which include 3, Ars Combin. 41 (1995), 129-161.

[25] H. Hanani, Balanced incomplete block designs and related designs, Discrete Math. 11 (1975), 255-369.

[26] D. R. Hughes and F. C. Piper, Design Theory, Cambridge University Press, Cambridge UK, (1985).

[27] Z. Janko and V. D. Tonchev, New designs with block size 7, J. Combin. Theory Ser. A 83 (1998), 152-157.

[28] E. Lamken, The existence of 3 orthogonal partitioned incomplete latin squares of type $t^{n}$, Discrete Math. 89 (1991), 231-251.

[29] A.C.H. Ling, Pairwise Balanced Designs and Related Codes, Ph. D. Thesis, University of Waterloo, Canada, 1997.

[30] A. C. H. Ling and C. J. Colbourn, Pairwise balanced designs with block sizes 8, 9 and 10, J. Combin. Theory Ser. A 77 (1997), 228-245.

[31] A.C.H. Ling and C. J. Colbourn, Concerning the generating set of $Z_{\geq n}$, Congr. Numer. 114 (1996), 65-72.

[32] A. C. H. Ling, X. J. Zhu, C. J. Colbourn and R. C. Mullin, Pairwise balanced designs with consecutive block sizes, Des. Codes Cryptogr. 10 (1997), 203-222.

[33] P. Lorimer, A class of block designs having the same parameters as the design of points and lines in a projective 3-space, Lecture Notes in Math. 403 (1974), 73-78.

[34] R. C. MacNeish, Euler squares, Ann. of Math. 23 (1922), 221-227.

[35] R.C. Mullin, Finite bases for some PBD-closed sets, Discrete Math. 77 (1989), $217-236$.

[36] R. C. Mullin, D. G. Hoffman and C. C. Lindner, A few more BIBD's with $k=6$ and $\lambda=1$, Ann. Discrete Math. 34 (1987), 379-384.

[37] R. C. Mullin, A. C.H. Ling, R. J. R. Abel and F.E. Bennett, On the closure of subsets of $\{4,5, \ldots, 9\}$ which contain 4, Ars Combin. 45 (1997), 33-76. 
THE ELECTRONIC JOURNAL OF COMBINATORICS 7 (2000), \#R13

[38] R. C. Mullin, P. J. Schellenberg, S. A. Vanstone and W. D. Wallis, On the existence of frames, Discrete Math. 37 (1981), 79-104.

[39] E. Seiden, A method of construction of resolvable BIBD, Sankhy a Ser. A 25 (1963), 393-394.

[40] R. M. Wilson, Concerning the number of mutually orthogonal latin squares, Discrete Math. 9 (1974), 181-198.

[41] R. M. Wilson, Constructions and uses of pairwise balanced designs, Math. Centre Tracts 55, Mathematisch Centrum, Amsterdam, 1974, 18-41.

\section{A Appendix}

The object of this appendix is to provide the constructions for the first 288 values of $B\left(Q_{1(8)}\right)$ plus portions of the range for the first 1648 values, as annonunced in Section 6 .

Key to Table A.1.

\begin{tabular}{|c|c|c|c|c|c|c|}
\hline Code & Result & Authority & Code & & Result & Authority \\
\hline $\mathrm{A} / \mathrm{a}$ & $17 m+n$ & Lemma 5.14 & $\mathrm{P}$ & & & Prime \\
\hline m.n & $17 m+n$ & Lemma 5.16 & $\mathrm{PP}$ & & & Prime power \\
\hline m.n & $10 m-n$ & Lemma 5.8 & $\mathrm{R}$ & v.n & $v+n$ & Lemma 5.10 \\
\hline m.n & $15 m+n$ & Lemma 5.17 & $\mathrm{RB}$ & $\mathrm{m}$ & $m$ & Lemma 6.2 \\
\hline $\mathrm{D} / \mathrm{d}$ & $17 m+n$ & Lemma 5.18 & $\mathrm{~T} / \mathrm{t}$ & m.n & $9 m+n$ & Lemma 5.5 \\
\hline m.n & $16 m+16+n$ & Lemma 5.19 & Table & & & Table 6.2 \\
\hline $\mathrm{m} . \mathrm{n}$ & $18 m-16+n$ & Lemma 5.21 & $\mathrm{X}$ & & & Tables $6.3,6.4$ \\
\hline m.n & $63 m+9+n$ & Lemma 5.23 & $\mathrm{ZZ}$ & & & Lemma 6.3 \\
\hline $\mathrm{m} . \mathrm{n}$ & $9 m+n-9$ & Lemma 5.7 & & & & \\
\hline m.n & $9 m+n-9$ & Lemma 5.6 & $* * \mathrm{n}$ & & Uncons & ucted \\
\hline
\end{tabular}

Lower case versions of construction codes (and their $m$ and $n$ ) are from Table 6.5.

Table A.1.

\begin{tabular}{|c|c|c|c|c|c|c|c|c|c|c|}
\hline & & & & Table & f Constr & ctions fo & $U_{1(8)}$ & & & \\
\hline & & 0 & 1 & 2 & 3 & 4 & 5 & 6 & 7 & 8 \\
\hline 0 & 0 & & PP & $\mathrm{P}$ & $\mathrm{PP}$ & $* * * 4 * *$ & $\mathrm{P}$ & PP & $* * * 7 * *$ & $* * * 8 * *$ \\
\hline 9 & 1 & $\mathrm{P}$ & PP & $\mathrm{P}$ & $\mathrm{P}$ & $* * 13 * *$ & $\mathrm{P}$ & PP & $* * 16^{* *}$ & $\mathrm{P}$ \\
\hline 18 & 2 & Table & Table & $* * 20^{* *}$ & PP & $* * 22 * *$ & $* * 23 * *$ & $\mathrm{P}$ & $* * 25 * *$ & $* * 26^{* *}$ \\
\hline 27 & 3 & $* * 27^{* *}$ & Table & $\mathrm{P}$ & $\mathrm{P}$ & $* * 31 * *$ & $\mathrm{P}$ & $* * 33^{* *}$ & Table & $\mathrm{P}$ \\
\hline 36 & 4 & PP & $* * 37^{* *}$ & $* * 38^{* *}$ & $\mathrm{P}$ & $* * 40 * *$ & RB 41 & $\mathrm{P}$ & $* * 43^{* *}$ & $\mathrm{P}$ \\
\hline 45 & 5 & $\mathrm{PP}$ & Table & $* * 47 * *$ & $* * 48^{* *}$ & $\mathrm{RB} 49$ & $\mathrm{P}$ & $\mathrm{P}$ & $* * 52^{* *}$ & Table \\
\hline 54 & 6 & $\mathrm{P}$ & Table & $\mathrm{P}$ & $\mathrm{P}$ & $* * 58 * *$ & $* * 59 * *$ & $* * 60 * *$ & $* * 61^{* *}$ & $* * 62^{* *}$ \\
\hline 63 & 7 & $* * 63^{* *}$ & Table & $\mathrm{P}$ & $\mathrm{PP}$ & $* * 67 * *$ & $* * 68^{* *}$ & $* * 69 * *$ & $* * 70^{* *}$ & $\mathrm{P}$ \\
\hline 72 & 8 & $\mathrm{P}$ & Table & $\mathrm{P}$ & $\mathrm{P}$ & $* * 76^{* *}$ & $\mathrm{P}$ & PP & $* * 79^{* *}$ & $\mathrm{P}$ \\
\hline 81 & 9 & Т 9.0 & Т 9.1 & Т 9.2 & $\mathrm{P}$ & $* * 85^{* *}$ & Т 9.5 & Т 9.6 & $* * 88^{* *}$ & $* * 89 * *$ \\
\hline 90 & 10 & Т 9.9 & PP & $* * 92^{* *}$ & $* * 93^{* *}$ & $* * 94^{* *}$ & $\mathrm{P}$ & $\mathrm{P}$ & RB 97 & $* * 98^{* *}$ \\
\hline 99 & 11 & T 11.0 & Т 11.1 & $\mathrm{P}$ & Т 11.3 & $* 103^{* *}$ & Т 11.5 & $\mathrm{PP}$ & $* 106^{* *}$ & $\mathrm{P}$ \\
\hline 108 & 12 & Т 11.9 & T 11.10 & $\mathrm{P}$ & $\mathrm{ZZ}$ & $* 112^{* *}$ & RB 113 & $* 114^{* *}$ & $* 115^{* *}$ & $\mathrm{P}$ \\
\hline 117 & 13 & $\mathrm{P}$ & $* 118^{* *}$ & $\mathrm{P}$ & $\mathrm{PP}$ & RB 121 & $\mathrm{P}$ & $* 123^{* *}$ & $* 124^{* *}$ & $* 125^{* *}$ \\
\hline
\end{tabular}




\begin{tabular}{|c|c|c|c|c|c|c|c|c|c|c|}
\hline 126 & 14 & $\mathrm{P}$ & Table & Table & $\mathrm{P}$ & $* 130 * *$ & $\mathrm{P}$ & $* 132 * *$ & $* 133^{* *}$ & $* 134^{* *}$ \\
\hline 135 & 15 & Table & Table & $\mathrm{P}$ & Table & $* 139 * *$ & $* 140 * *$ & $\mathrm{P}$ & $* 142 * *$ & $* 143^{* *}$ \\
\hline 144 & 16 & $\mathrm{P}$ & t 16.1 & t 16.2 & t 16.3 & $* 148^{* *}$ & $\mathrm{P}$ & $\mathrm{P}$ & $* 151^{* *}$ & $\mathrm{P}$ \\
\hline 153 & 17 & T 17.0 & $\mathrm{~T} 17.1$ & T 17.2 & $\mathrm{P}$ & * $157^{* *}$ & $\mathrm{~T} 17.5$ & T 17.6 & $* 160 * *$ & $\mathrm{P}$ \\
\hline 162 & 18 & $\mathrm{P}$ & Т 17.10 & Т 17.11 & $\mathrm{P}$ & $* 166^{* *}$ & Т 17.14 & Т 17.15 & RB 169 & $\mathrm{P}$ \\
\hline 171 & 19 & $\mathrm{PP}$ & Т 19.1 & T 19.2 & Т 19.3 & * $175^{* *}$ & $\mathrm{P}$ & Т 19.6 & $* 178^{* *}$ & $\mathrm{P}$ \\
\hline 180 & 20 & Т 19.9 & Т 19.10 & Т 19.11 & Т 19.12 & $* 184^{* *}$ & $\mathrm{P}$ & $\mathrm{P}$ & $* 187^{* *}$ & Т 19.17 \\
\hline 189 & 21 & Т 19.18 & Т 19.19 & $* 191^{* *}$ & $* 192^{* *}$ & RB 193 & $\mathrm{P}$ & $* 195^{* *}$ & $* 196^{* *}$ & $* 197^{* *}$ \\
\hline 198 & 22 & ZZ & $* 199 * *$ & $\mathrm{P}$ & $\mathrm{P}$ & $* 202^{* *}$ & $* 203^{* *}$ & $* 204^{* *}$ & $* 205^{* *}$ & Table \\
\hline 207 & 23 & $\mathrm{P}$ & $* 208^{* *}$ & *209** & $\mathrm{PP}$ & $* 211^{* *}$ & $\mathrm{P}$ & $* 213^{* *}$ & $* 214^{* *}$ & $\mathrm{P}$ \\
\hline 216 & 24 & Table & Table & $* 218^{* *}$ & $\mathrm{P}$ & $* 220 * *$ & $* 221^{* *}$ & $\mathrm{P}$ & $* 223^{* *}$ & $* 224^{* *}$ \\
\hline 225 & 25 & $\mathrm{P}$ & *226** & $* 227 * *$ & t 25.3 & $* 229 * *$ & t 25.5 & $\mathrm{PP}$ & $* 232^{* *}$ & RB 233 \\
\hline 234 & 26 & $\mathrm{P}$ & t 25.10 & $\mathrm{P}$ & t 25.12 & $* 238^{* *}$ & $\mathrm{P}$ & t 25.15 & RB 241 & t 25.17 \\
\hline 243 & 27 & t 25.18 & t 27.1 & t 27.2 & t 27.3 & $* 247 * *$ & t 27.5 & $\mathrm{P}$ & $* 250 * *$ & Table \\
\hline 252 & 28 & $\mathrm{P}$ & t 27.10 & t 27.11 & t 27.12 & $* 256 * *$ & t 27.14 & t 27.15 & $* 259 * *$ & $\mathrm{P}$ \\
\hline 261 & 29 & $\mathrm{P}$ & Т 29.1 & Т 29.2 & $\mathrm{P}$ & $* 265^{* *}$ & $\mathrm{P}$ & $\mathrm{P}$ & $* 268^{* *}$ & $\mathrm{P}$ \\
\hline 270 & 30 & $\mathrm{P}$ & Т 29.10 & Т 29.11 & Т 29.12 & a 16.2 & Т 29.14 & $\mathrm{PP}$ & a 16.5 & Т 29.17 \\
\hline 279 & 31 & Т 29.18 & Т 29.19 & a 16.9 & Т 29.21 & a 16.11 & $\mathrm{P}$ & $\mathrm{P}$ & a 16.14 & $\mathrm{P}$ \\
\hline 288 & 32 & Т 32.0 & $\mathrm{X}$ & $\mathrm{X}$ & $\mathrm{X}$ & $\mathrm{X}$ & $\mathrm{X}$ & $\mathrm{X}$ & $\mathrm{X}$ & $\mathrm{X}$ \\
\hline \multicolumn{11}{|c|}{ For 289-321; use construction A with $m=17$. } \\
\hline 15 & 35 & $\mathrm{X}$ & $\mathrm{X}$ & $\mathrm{X}$ & $\mathrm{X}$ & $\mathrm{X}$ & $\mathrm{X}$ & $\mathrm{X}$ & t 32.34 & $\mathrm{X}$ \\
\hline 324 & 36 & a 17.35 & $\mathrm{X}$ & $\mathrm{P}$ & $\mathrm{X}$ & $\mathrm{X}$ & В 19.6 & $\mathrm{X}$ & В 19.8 & $\mathrm{X}$ \\
\hline
\end{tabular}

For $323-357$ (with gaps); use construction $\mathrm{C}$ with $m=19$.

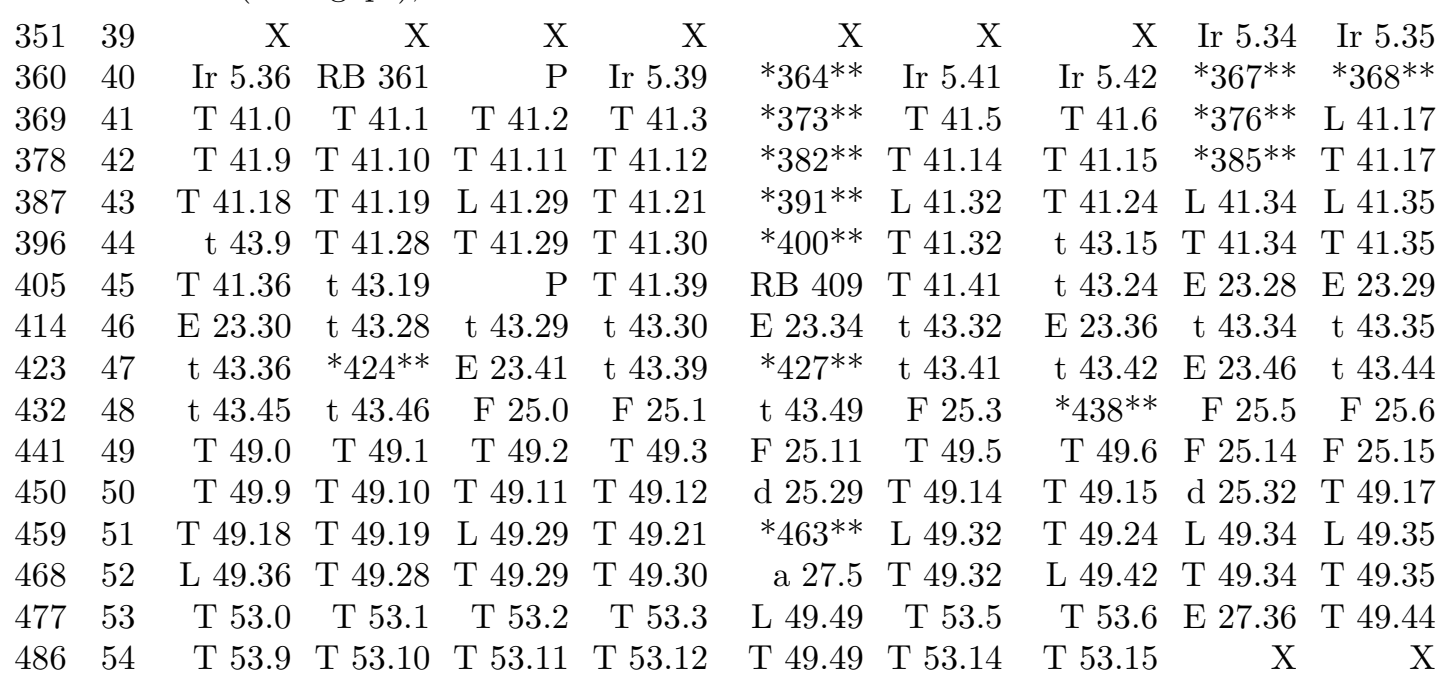
For 493-606, 608; use constructions A, C with $m=29,32$.

\begin{tabular}{|c|c|c|c|c|c|c|c|c|c|}
\hline 3 & & & $\mathrm{X}$ & X & & & & & \\
\hline 2 & Т 64.36 & R585.28 & R585.29 & Т 64.39 & Ls64.49 & & & & \\
\hline & & & ${ }^{*} 623 * *$ & & 9 & & & & \\
\hline & & & Т 6 & & & & & & \\
\hline & & & & & $* 64$ & & & & \\
\hline & & & $\mathrm{T} 7$ & & & & & & \\
\hline & & & & & & & & & \\
\hline & & & & & & & & & \\
\hline & & & & & & & & & \\
\hline & & & & & 11.49 & 11.50 & Г 71.51 & 73.34 & \\
\hline
\end{tabular}




\begin{tabular}{|c|c|c|c|c|c|c|c|c|c|c|}
\hline 693 & 77 & T 71.54 & T 71.55 & Т 71.56 & T 71.57 & $\mathrm{X}$ & $\mathrm{X}$ & $\mathrm{X}$ & $\mathrm{X}$ & $x$ \\
\hline \multicolumn{11}{|c|}{ For $697-779$; use constructions A, C with $m=41$. } \\
\hline 774 & 86 & $\mathrm{X}$ & $\mathrm{X}$ & $\mathrm{X}$ & $\mathrm{X}$ & $\mathrm{X}$ & $\mathrm{X}$ & T 81.51 & T 83.34 & \\
\hline 783 & 87 & $\mathrm{~T} 81.54$ & $\mathrm{~T} 81.55$ & T 81.56 & T 81.57 & Т 82.49 & T 82.50 & 2.51 & 3.32 & \\
\hline 792 & 88 & $\mathrm{~T} 82.54$ & $\mathrm{~T} 81.64$ & T 81.65 & Т 81.66 & Т 83.49 & T 83.50 & T 83.51 & F 43.41 & T 8 \\
\hline 801 & 89 & Т 81.72 & $\mathrm{~T} 81.73$ & $\mathrm{~T} 81.74$ & T 81.75 & ZZ & T 81.77 & T 81.78 & c 43.82 & 1.80 \\
\hline 810 & 90 & Т 81.81 & Т 82.73 & Т 82.74 & Т 82.75 & ZZ & T 82.77 & Т 82.78 & c 43.91 & T 82.80 \\
\hline 819 & 91 & Т 82.81 & Т 82.82 & $\mathrm{~T} 83.74$ & T 83.75 & $* 823^{* *}$ & T 83.77 & T 83.78 & ZZ & Т 83.80 \\
\hline 828 & 92 & Т 83.81 & Т 83.82 & T 83.83 & t 83.84 & $* 832 * *$ & $\mathrm{X}$ & $\mathrm{X}$ & $\mathrm{X}$ & \\
\hline
\end{tabular}

For 833-1006; use construction $\mathrm{C}$ with $m=49,53$.

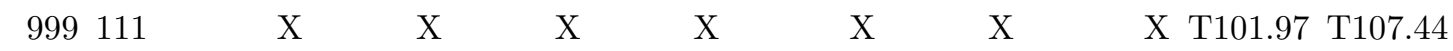

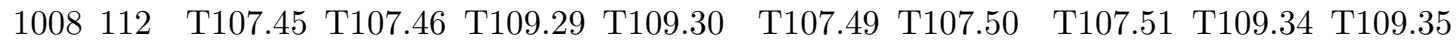

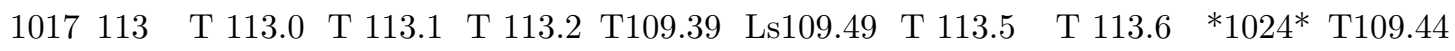

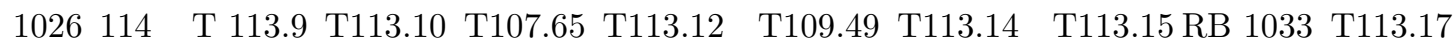

1035115 T113.18 T113.19 T109.56 T113.21 *1039* T107.77 T113.24 L113.34 T107.80

1044116 T107.81 T109.64 T113.29 T113.30 *1048* T113.32 $\quad$ T107.87 T113.34 T113.35

$\begin{array}{llllllll}1053 & 117 & \text { T113.36 } & \text { T109.73 } & \text { T109.74 T113.39 } & \text { L113.49 T113.41 } & \text { T113.42 } & \text { T107.97 T113.44 }\end{array}$

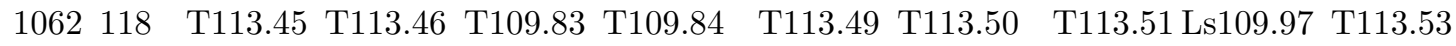

$\begin{array}{llllllll}1071 & 119 & \text { T113.54 T113.55 T113.56 T113.57 } & \text { Ir15.121 T109.95 } & \text { T109.96 T109.97 L113.71 }\end{array}$

1080120 T109.99 T113.64 T113.65 T113.66 *1084* T117.32 T109.105 T117.34 X

For 1088-1575; use constructions A, C with $m=64,71,73,81,83$.

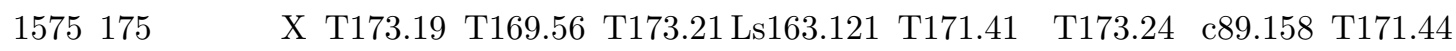

$\begin{array}{lllllllllll}1584 & 176 & \text { T } 176.0 & \text { T } 176.1 & \text { T } 176.2 & \text { T } 176.3 & \text { T171.49 } & \text { T } 176.5 & \text { T } 176.6 & \text { T173.34 } & \text { T173.35 }\end{array}$

$\begin{array}{lllllllllll}1593 & 177 & \text { T } 177.0 & \text { T } 177.1 & \text { T } 177.2 & \text { T } 177.3 & \text { Ls173.49 } & \text { T } 177.5 & \text { T } 177.6 & \text { T167.97 } & \text { T176.17 }\end{array}$

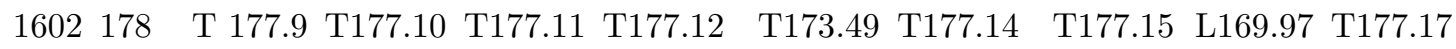

$\begin{array}{lllllllll}1611 & 179 & \text { T } 179.0 & \text { T } 179.1 & \text { T } 179.2 & \text { T } 179.3 \text { Ls167.121 } & \text { T } 179.5 & \text { T } 179.6 & \text { T176.34 T176.35 }\end{array}$

$\begin{array}{lllllllll}1620 & 180 & \text { T } 179.9 & \text { T179.10 T179.11 T179.12 T167.121 T179.14 } & \text { T179.15 T177.34 T179.17 }\end{array}$

$\begin{array}{lllllllllll}1629 & 181 & \text { T } 181.0 & \text { T } 181.1 & \text { T } 181.2 & \text { T } 181.3 & \text { T176.49 } & \text { T } 181.5 & \text { T } 181.6 & \text { T171.97 } & \text { T177.44 }\end{array}$

$\begin{array}{llllllll}1638 & 182 & \text { T } 181.9 & \text { T181.10 T181.11 T181.12 } & \text { T177.49 } & \text { T181.14 } & \text { T181.15 T179.34 T181.17 }\end{array}$

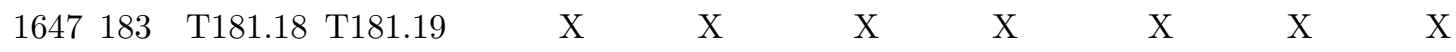

$\begin{array}{lllllllll}0 & 1 & 2 & 3 & 4 & 5 & 6 & 7 & 8\end{array}$

